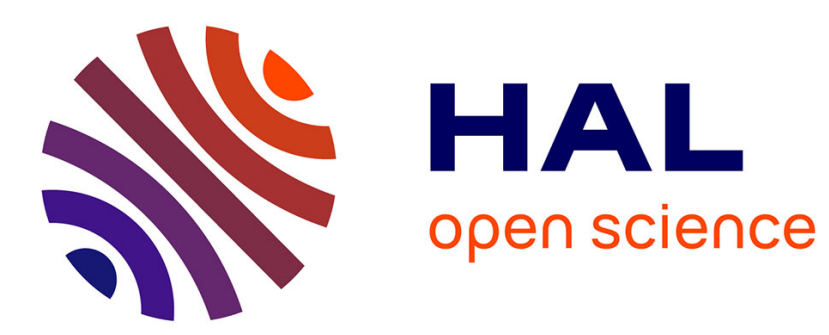

\title{
Implementation of an urban canopy parameterization in a mesoscale meteorological model
}

Tanya L. Otte, Avraham Lacser, Sylvain Dupont, Jason K.S. Ching

\section{To cite this version:}

Tanya L. Otte, Avraham Lacser, Sylvain Dupont, Jason K.S. Ching. Implementation of an urban canopy parameterization in a mesoscale meteorological model. Journal of Applied Meteorology, 2004, 43 (11), pp.1648-1665. 10.1175/JAM2164.1 . hal-02683339

\section{HAL Id: hal-02683339 \\ https://hal.inrae.fr/hal-02683339}

Submitted on 1 Jun 2020

HAL is a multi-disciplinary open access archive for the deposit and dissemination of scientific research documents, whether they are published or not. The documents may come from teaching and research institutions in France or abroad, or from public or private research centers.
L'archive ouverte pluridisciplinaire HAL, est destinée au dépôt et à la diffusion de documents scientifiques de niveau recherche, publiés ou non, émanant des établissements d'enseignement et de recherche français ou étrangers, des laboratoires publics ou privés. 


\title{
Implementation of an Urban Canopy Parameterization in a Mesoscale Meteorological Model
}

\author{
TANYA L. OTTE* \\ Atmospheric Sciences Modeling Division, Air Resources Laboratory, National Oceanic and Atmospheric Administration, \\ Research Triangle Park, North Carolina \\ AVRAHAM LACSER \\ Israel Institute for Biological Research, Ness Ziona, Israel \\ Sylvain Dupont ${ }^{*}+$ AND Jason K. S. Ching ${ }^{*}$ \\ Atmospheric Sciences Modeling Division, Air Resources Laboratory, National Oceanic and Atmospheric Administration, \\ Research Triangle Park, North Carolina
}

(Manuscript received 23 May 2003, in final form 8 March 2004)

\begin{abstract}
An urban canopy parameterization (UCP) is implemented into the fifth-generation Pennsylvania State University-National Center for Atmospheric Research Mesoscale Model (MM5) to improve meteorological fields in the urban boundary layer for finescale $(\sim 1-\mathrm{km}$ horizontal grid spacing) simulations. The UCP uses the dragforce approach for dynamics and a simple treatment of the urban thermodynamics to account for the effects of the urban environment. The UCP is evaluated using a real-data application for Philadelphia, Pennsylvania. The simulations show that the UCP produces profiles of wind speed, friction velocity, turbulent kinetic energy, and potential temperature that are more consistent with the observations taken in urban areas and data from idealized wind tunnel studies of urban areas than do simulations that use the roughness approach. In addition, comparisons with meteorological measurements show that the UCP simulations are superior to those that use the roughness approach. This improvement of the treatment of the urban areas in the meteorological model could have implications for simulating air chemistry processes at this scale.
\end{abstract}

\section{Introduction}

The meteorological fields in the urban boundary layer at finescales result from the interactions between the larger-scale meteorological fields and the urban surfaces. The fabric of the urban canopy (i.e., the layer from the surface to the tops of buildings) is very heterogeneous. Therefore, the meteorological fields can be very complex in the urban roughness sublayer (URSL), that is, the layer from the surface to the height at which the effects of the individual surface roughness elements on the flow cannot be distinguished, or approximately 25 times the depth of the urban canopy layer (Rotach

\footnotetext{
* On assignment to the National Exposure Research Laboratory, U.S. Environmental Protection Agency, Research Triangle Park, North Carolina.

+ Current affiliation: INRA-EPHYSE, Villenauve d'Ornon, France.
}

Corresponding author address: Tanya L. Otte, EPA/NERL/AMD, Mail Drop E243-03, Research Triangle Park, NC 27711.

E-mail: otte.tanya@epa.gov
1999). Given the current computer capacity, it may be impractical to use sufficiently fine grid spacing in a mesoscale model to simulate explicitly the flows around the individual surface obstacles (e.g., buildings and trees) for an entire city and its surrounding area. However, to assess human exposure to air pollutants in urban areas, it is necessary to generate detailed meteorological fields inside the URSL since primary atmospheric pollutants are dispersed within this layer.

The aerodynamic characteristics of the urban surface are often represented in mesoscale models as simplified parameterizations by applying the roughness approach, in which a gridded roughness length and a displacement height are used to represent the influences of the surface obstacles on the flow. In the roughness approach, the dynamic and thermodynamic surface exchange coefficients are calculated from the Monin-Obukhov similarity theory, which assumes stationary conditions and spatial homogeneity. As explained by Martilli (2002), these assumptions break down in the URSL. Furthermore, the roughness approach cannot simulate the thermodynamic profiles below the displacement height and 


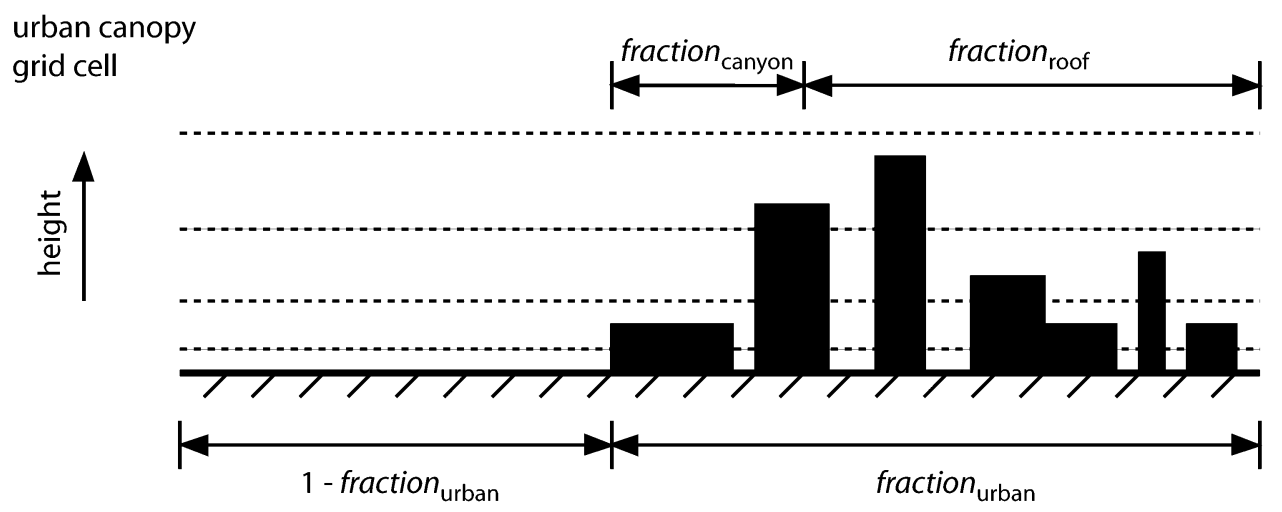

FIG. 1. Schematic illustration of side view of an urban gridcell representation in the model (adapted from Brown and Williams 1998). The grid cell is partitioned into area defined as urban and nonurban. The canyon regions are defined in the areas between buildings, and the sum of the canyon areas is $f_{\text {cnyn }}$. The remainder of $f_{\text {urb }}$ is defined as $f_{\text {roof }}$. There are several model layers (shown as dashed lines) within the urban canopy layer.

does not reproduce the turbulent kinetic energy (TKE) maximum observed above the urban canopy. Thus, the roughness approach may be inappropriate for simulating the meteorological fields with a mesoscale model at finescales (i.e., $\sim 1-\mathrm{km}$ horizontal grid spacing) in urban areas.

To improve the mesoscale model simulation of meteorological fields inside the URSL, the drag-force approach that was used for the vegetation canopy (Yamada 1982) has recently been extended to the urban canopy (cf. Brown and Williams 1998; Martilli et al. 2002). In comparison with the roughness approach, the lowest level of the computational domain in the drag-force approach corresponds to the real level of the ground (no displacement height). Some vertical layers are added within the urban canopy to allow for the simulation of detailed meteorological fields in the URSL (see Fig. 1). Inside the urban canopy, the gridcell-averaged effect of the building structures on the dynamics and thermodynamics is parameterized following the local characteristics of the urban morphology; individual buildings and street canyons are not explicitly represented.

In this study, an urban canopy parameterization (UCP) is introduced into the fifth-generation Pennsylvania State University-National Center for Atmospheric Research Mesoscale Model (MM5; Grell et al. 1994). This study is designed to compare the modeled fields from the drag-force approach and the roughness approach for an urban area and its surroundings. The UCP treats the dynamic effects of the urban areas on the flow following the drag-force approach described by Brown and Williams (1998) and Brown (2000). The dynamic aspect of the UCP accounts for the drag exerted by the urban structures and the enhancement of TKE, especially near the tops of buildings. Because the focus of this study is on demonstrating the impact of the dragforce approach in MM5, a simplified treatment of the urban thermodynamics is used. Here, the thermodynamic aspect of the UCP includes the modification of the surface energy budget because of shadowing and trapping of radiation between buildings, and the three-dimensional energy contribution from radiation between buildings, at rooftops, and from anthropogenic sources. A more sophisticated version of the UCP with advanced thermodynamics following Dupont (2001) will be presented in a forthcoming paper.

In this paper, we discuss the formulation and implementation of the UCP in MM5, as well as its evaluation using a real-data case on a $1.33-\mathrm{km}$ domain centered on Philadelphia, Pennsylvania. Section 2 contains a description of the UCP formulation. Section 3 describes the configuration of MM5 and the UCP for the Philadelphia case. Section 4 contains the results of simulations with the UCP and comparisons with simulations using the roughness approach. Section 5 provides conclusions and implications for future work.

\section{Urban canopy parameterization formulation}

The UCP accounts for dynamic and thermodynamic effects on the flow caused by the presence of urban structures. In the UCP, the fraction of urban area in each grid cell of the domain $\left(f_{\text {urb }}\right)$ is partitioned into total area represented by canyons (i.e., street level between buildings, $\left.f_{\text {cnyn }}\right)$ and roofs $\left(f_{\text {roof }}\right)$ from the planar view of the city (see Fig. 1). In this formulation, it is assumed that the buildings affect the flow, virtually, because of their horizontal and vertical surfaces. Analogous to the model used for the vegetation canopy, we assume that buildings do not take up any volume within the grid cell; other models for the urban area (cf. Martilli 2002) consider the volume of buildings through the calculation of the vertical turbulent fluxes. The vertical resolution in the meteorological model is increased so that there are several prognostic layers within the urban canopy. In our implementation, the drag-force approach is used to represent the dynamic effects of buildings. The effects of the vegetation and bare soil are represented by using 
the roughness approach. Outside the urban area, the roughness approach is applied with a thinner first vertical layer than in a typical application of MM5, and the assumption of a constant flux layer considered by Monin-Obukhov similarity theory may be less applicable for the thin layer.

\section{a. Effects on dynamics}

The horizontal components of the momentum and TKE equations are modified to account for the areaaverage effect of the subgrid urban elements following Brown (2000). These changes consider the drag exerted by the urban structures and the enhancement of TKE, especially near the tops of buildings, and they are targeted for a planetary boundary layer (PBL) parameterization scheme that has a prognostic equation for TKE. Here, the momentum and TKE modifications are implemented in MM5 through the TKE-based Gayno-Seaman PBL (GSPBL) scheme (e.g., Shafran et al. 2000).

The momentum equations accounting for the urban elements are

$$
\begin{aligned}
\frac{\partial U}{\partial t} & =F_{U}-0.5 f_{\mathrm{urb}} C_{d} A_{f}(z) U\left(U^{2}+V^{2}\right)^{0.5}, \\
\frac{\partial V}{\partial t} & =F_{V}-0.5 f_{\mathrm{urb}} C_{d} A_{f}(z) V\left(U^{2}+V^{2}\right)^{0.5}, \quad \text { and } \\
\frac{\partial \mathrm{TKE}}{\partial t} & =F_{\mathrm{TKE}}+0.5 f_{\mathrm{urb}} C_{d} A_{f}(z)\left(U^{2}+V^{2}+W^{2}\right)^{1.5},
\end{aligned}
$$

where $F_{U}, F_{V}$, and $F_{\text {TKE }}$ are the model's general forcing terms in each equation; $U, V$, and $W$ are the mean wind components; $C_{d}$ is the canopy drag coefficient; and $A_{f}(z)$ is the canopy area density, or the surface area of the obstacles (e.g., buildings) perpendicular to the wind per unit volume of the urban canopy, expressed in meters squared per meters cubed. The drag term in the modified momentum Eqs. (1) and (2) follows from the formulations given in Byun and Arya (1986) and Uno et al. (1989), for example. The new terms in Eqs. (1) and (2) are solved in the UCP using the analytical solution suggested by Byun and Arya (1986). Since TKE is composed of all three wind components, $W$ is also included in the drag term in Eq. (3) (cf. Maruyama 1999), although its value is small in comparison with $U$ and $V$. The TKE [Eq. (3)] is solved explicitly in the UCP.

Here $A_{f}(z)$ is defined such that it is maximized within the urban canopy and vanishes at the top of the urban canopy so that the drag term also vanishes at that level (e.g., Uno et al. 1989; Brown 2000). The integral of $A_{f}(z)$ from the surface to the height of the urban canopy $h_{c}$ is $\lambda_{f}$, which corresponds to the ratio of the frontal area of buildings to the total urban surface area. In the absence of a detailed morphology database, $A_{f}(z)$ can be estimated from $\lambda_{f}$ and $h_{c}$ by assuming a functional form for $A_{f}(z)$.

The drag on the wind speed and the production of TKE are linear functions of the drag coefficient $C_{d}$, which depends on the shape and distribution of buildings within a grid cell. It is intuitive that $C_{d}$ is smaller where buildings are closely spaced and of similar height and that it is larger where there is more variation in the height and spacing of buildings. Brown (2000) suggests that there is still great uncertainty in the specific setting of $C_{d}$. Brown and Williams (1998) suggest values for $C_{d}$ ranging from 0.7 to 1.5 for urban structures. For simplicity, $C_{d}$ is assumed to be constant in the vertical direction (e.g., Brown and Williams 1998; Martilli et al. 2002), and it is set to 1.0 for all urban areas. The effects of building density on the drag are included in Eqs. (1), (2), and (3) through $A_{f}(z)$.

We note that the large eddies above the urban canopy will break when they come in contact with the urban structures, thus creating different turbulent length scales in the URSL within and above the urban canopy. For completeness, the turbulent length scale should also be modified within the urban canopy as part of the UCP. However, for simplicity, the turbulent length scales are equivalent in the URSL in this version of the UCP.

\section{b. Effects on thermodynamics}

The effects of the urban environment on the thermodynamics are captured in the UCP using simplified approximations in the air temperature tendency equation and the ground surface energy budget. These urban effects account for the modification of the surface energy budget by shadowing and trapping of radiation between buildings and for the three-dimensional energy contribution from radiation between buildings, at rooftops, and from anthropogenic sources. In this implementation, the effects of water runoff from urban surfaces are ignored.

The thermodynamic equations for the UCP adapted from Brown and Williams (1998) are

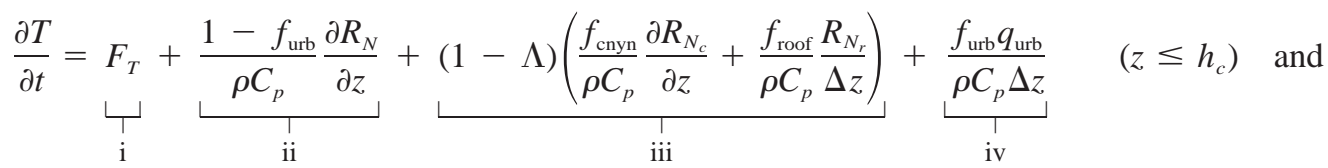

$$
\begin{aligned}
& \frac{\partial T}{\partial t}=F_{T}+\frac{1}{\rho C_{p}} \frac{\partial R_{N}}{\partial z} \quad\left(z>h_{c}\right)
\end{aligned}
$$


where $\rho$ is the air density and $C_{p}$ is the specific heat of dry air.

Inside the canopy, the temperature equation [Eq. (4a)] accounts for (i) thermal forcing from all physical processes excluding radiation $\left(F_{T}\right)$, (ii) general radiative forcing unrelated to the urban environment, where $R_{N}$ represents the net radiation, (iii) thermal effects due to urban structures, and (iv) thermal effects due to anthropogenic sources. Equation (4b) is MM5's general equation for temperature above the canopy and in nonurban areas with the radiative contributions explicitly shown for analogy with Eq. (4a).

In term iii of Eq. (4a), $\Lambda$ represents the heat storage in the urban area defined by the ratio of the storage heat flux to the net radiation [e.g., $\Delta Q_{s} / Q^{*}$, following notation by Grimmond and Oke (1999a)]. We use $\Lambda=$ 0.4 here, which is within the range of measurements for urban areas reported by Grimmond and Oke (1999a). For artificial surfaces (e.g., pavement, roofs, walls), the storage heat flux in the energy budget of these surfaces cannot be neglected in comparison with the sensible heat flux. The storage heat flux can sometimes exceed the sensible heat flux (e.g., Grimmond and Oke 1999a). However, if there is no precipitation, the latent heat flux is negligible. Thus, by neglecting the latent heat flux from urban surfaces, $1-\Lambda$ represents the ratio between the sensible heat flux and the net radiation flux. Thus, the first half of term iii in Eq. (4a) represents the sensible heat flux from the street canyons at the level $z$ within the canopy, which could be attributed to the sensible heat flux from building walls. The second half of term iii in Eq. (4a) represents the sensible heat flux from roofs, where $R_{N_{r}}$ is the roof net radiation.

To consider the effects of shadowing and trapping of radiation in the urban canyons, we use an extinction method similar to what has been done for vegetation canopies. In the first part of term iii in Eq. (4a), $R_{N_{c}}$ is the in-canopy net radiation considering the shadowing and trapping of radiation in the urban canyon (i.e., between buildings). It is defined using an exponential function following Yamada (1982):

$$
R_{N_{c}}=R_{N_{h}} e^{-k L(z)},
$$

where $R_{N_{h}}$ is the net radiation at the top of the urban canopy $h_{c}$ (i.e., top of the canyon). The canyon radiation extinction coefficient $k$ represents the modulation of the cumulative canopy area density in the exponential function. In the absence of data specific to an urban canyon, we use a value of 0.6, which Yamada (1982) used for radiation extinction in a vegetation canopy.

In Eq. (5), $L(z)$ is the cumulative building area density, which is analogous to leaf area index in the vegetation model. Here $L(z)$ is evaluated from height $z$ within the canopy to $h_{c}$, given by

$$
L(z)=\int_{z}^{h_{c}} A_{p}\left(z^{\prime}\right) d z^{\prime} .
$$

Here $A_{p}(z)$ is the building plan area density, or the surface area of the building floor space or roofs at a given height per unit volume of the urban canopy $\left(\mathrm{m}^{2} \mathrm{~m}^{-3}\right)$. The building area density at the surface, $L(0)$, is simply $\lambda_{p}$, which is the ratio of the building plan area to the total urban surface area.

The second part of term iii in Eq. (4a) is the contribution to the urban energy budget by the heat emitted by rooftops. It is assumed that the rooftops store the same amount of energy following the net radiation as the street canyons, that is, the same $\Lambda$. The rooftop net radiation $R_{N_{r}}$ is calculated from

$$
R_{N_{r}}=\left(1-\alpha_{\text {roof }}\right) R_{S \downarrow}+\Delta R_{L_{h_{c}}}-\varepsilon_{\text {roof }} \sigma T_{\text {roof }}^{4},
$$

where the albedo $\left(\alpha_{\text {roof }}\right)$, emissivity $\left(\varepsilon_{\text {roof }}\right)$, temperature $\left(T_{\text {roof }}\right)$, downward shortwave radiation $\left(R_{S \downarrow}\right)$, and net longwave radiation $\left(\Delta R_{L_{h_{c}}}\right)$ are defined at the rooftops, and $\sigma$ is the Stefan-Boltzmann constant.

In our implementation, we assume all rooftops are flat; we do not consider the angle and orientation of rooftops (e.g., pitches on suburban residential houses). Brown (2000) uses $T_{\text {air }}$ as a surrogate for $T_{\text {roof }}$ but suggests that directly solving the temperature of the rooftops is more appropriate. We assume that the rooftop's longwave emission radiates at the ground surface temperature, and so for simplicity we use the ground temperature $\left(T_{\text {sfc }}\right)$ as a surrogate for $T_{\text {roof }}$, because $T_{\text {sfc }}$ has a large diurnal amplitude as might be expected with rooftop temperatures. Although Voogt and Grimmond (2000) note that there can be large differences between $T_{\text {roof }}$ and $T_{\text {sfc }}$ (e.g., up to $\sim 10 \mathrm{~K}$ ), they can sometimes be explained by the transpiration of vegetation at the surface, which does not also occur at rooftops. In our simulations, a small moisture availability (0.10; Grell et al. 1994) is used for all urban areas, and so the effects of transpiration on the temperature difference should be minimized. The values of $T_{\mathrm{sfc}}$ in the UCP simulations are consistent with $T_{\text {roof }}$ values measured by Voogt and Grimmond (2000) for a summer case. We recognize that using $T_{\text {sfc }}$ to define $T_{\text {roof }}$ for the computation of the rooftop longwave radiation is generally not a valid assumption. However, for some cases (particularly dry cases with minimal vegetation on clear days, as we have modeled in our study), neglecting the difference between $T_{\text {roof }}$ and $T_{\text {sfc }}$ will have a very small impact (on average, about $5 \mathrm{~W} \mathrm{~m}^{-2}$ for a $5-\mathrm{K}$ error in our case); in other cases, it is not as appropriate. Although we do not advocate this simplification for universal use, for our initial study this assumption seems reasonable. Note that Chin et al. (2000) suggest a more complete method for assessing the energy budget at the rooftops, and they determine $T_{\text {roof }}$ by using a roof surface energy equation.

Term iv in Eq. (4a) is the contribution to the urban heat budget from anthropogenic sources (e.g., vehicles and mechanical heat from buildings). The anthropogenic heat flux is difficult to estimate and is somewhat controversial. Pielke (1984) suggests a value of $100 \mathrm{~W} \mathrm{~m}^{-2}$ for heat input in an urban area by anthropogenic and 


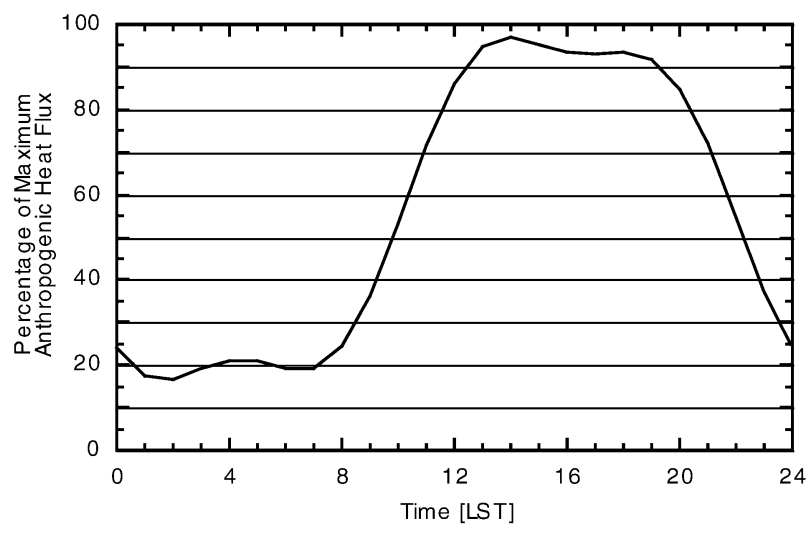

FIG. 2. Diurnal function for anthropogenic heat contribution as percentage of maximum anthropogenic heat flux. See also Sailor (1995).

natural sources. Grimmond and Oke (1995) suggest peak suburban values of anthropogenic heat flux on the order of $20 \mathrm{~W} \mathrm{~m}^{-2}$ in the daytime and $10 \mathrm{~W} \mathrm{~m}^{-2}$ overnight. Sailor and Fan (2002) suggest that the contribution from anthropogenic heating in urban areas is often on the order of $10-20 \mathrm{~W} \mathrm{~m}^{-2}$ but can be larger in the urban core. Masson (2000), however, suggests that the anthropogenic heat fluxes in urban areas can be much higher; his calculations for a cold-climate location generated values of $200 \mathrm{~W} \mathrm{~m}^{-2}$ or more in winter, largely predicated on domestic heating. Masson (2000) notes that anthropogenic heat fluxes for Montreal, Canada, can range from $153 \mathrm{~W} \mathrm{~m}^{-2}$ in winter to $57 \mathrm{~W} \mathrm{~m}^{-2}$ in summer and that the larger urban areas of Moscow, Russia, and Manhattan, New York, can be $25 \%$ and $50 \%$ greater, respectively.

In this UCP, the anthropogenic heat flux is included in the air temperature equation rather than in the surface energy budget (e.g., Chin et al. 2000) because the heat is released to the air. We use a time-varying anthropogenic heat flux $q_{\text {urb }}$ that is defined following Taha (1999) as

$$
q_{\mathrm{urb}}=\left(Q_{\text {Amax }}\right) F(t)
$$

Here $Q_{\text {Amax }}$ is the maximum anthropogenic heat flux, which is modulated diurnally by the Fourier series $F(t)$ :

$F(t)=\gamma+\sum_{n=1}^{3}\left[\lambda_{n} \cos \left(\frac{2 n \pi t}{24}\right)+\phi_{n} \sin \left(\frac{2 n \pi t}{24}\right)\right]$.

Following Taha (1999), $\gamma=0.557, \lambda_{1}=-0.227, \lambda_{2}$ $=-0.006, \lambda_{3}=-0.084, \phi_{1}=-0.384, \phi_{2}=0.016$, and $\phi_{3}=-0.012$. This series, also used in a mesoscale model by Sailor (1995), represents the summertime anthropogenic heating profile deduced from energy studies of various cities, and it is maximized during daytime. The effect of the anthropogenic heat flux is distributed through the depth of the urban canopy using the profile $A_{p}(z)$. Figure 2 shows the diurnal pattern for $q_{\text {urb }}$ as a percentage of the maximum anthropogenic heat flux.
The anthropogenic heat flux approaches but does not reach the prescribed maximum value $Q_{\text {Amax }}$. Using this function, the average hourly contribution from the anthropogenic heat flux is about $55 \%$ of the maximum value. Within the urban area, $q_{\text {urb }}$ may actually vary as a function of the surroundings as well as of the difference between the air temperature and various surface temperatures of the building, and those effects are not captured with this function.

Last, the UCP includes changes to the urban surface energy balance caused by thermodynamic effects in urban canyons. The changes to the urban surface energy balance are implemented in MM5 in the force-restore "slab" surface model (Zhang and Anthes 1982) through the equation for ground net radiation $R_{N_{G}}$ :

$$
\begin{aligned}
R_{N_{G}}= & \left(1-f_{\text {urb }}\right)\left[\left(1-\alpha_{G}\right) S_{G}+\Delta L_{G}\right] \\
& +f_{\text {cnyn }} R_{N_{h}} e^{-k L(0)}
\end{aligned}
$$

where $\alpha_{G}$ is the surface albedo, $S_{G}$ is the downward shortwave radiation at the ground, $\Delta L_{G}$ is the net longwave radiation at the ground, and the other terms are as defined earlier. The first term of Eq. 10 refers to the mesoscale model's unmodified shortwave and longwave energy contributions to the radiation budget at the surface in nonurban areas. The second term includes the shadowing and trapping effects of the net radiation reaching the ground modified by the extinction of radiation through the urban canopy using the ground cumulative building area density [i.e., $L(0)$ is deduced from Eq. (6) evaluated at the ground level]. Note that there is no contribution to the surface energy balance from the fraction of the urban area represented by $f_{\text {roof }}$.

\section{Configuring the model for Philadelphia, Pennsylvania}

\section{a. MM5 setup}

The nonhydrostatic MM5, version 3, release 5 (MM5v3.5), is run in a one-way-nested configuration for several days in July of 1995. The five nested MM5 computational domains include 108-, 36-, 12-, 4-, and $1.33-\mathrm{km}$ horizontal grid spacing. The first four domains are run with 30 vertical (terrain-following "sigma") layers (about 12 layers in the PBL and lowest-layer depth of $38 \mathrm{~m}$ ) and physics options appropriate for each resolution. Multiscale four-dimensional data assimilation (FDDA) is used on those four domains as in Stauffer and Seaman (1994).

The $1.33-\mathrm{km}$ domain includes $112 \times 112$ grid points covering the Philadelphia metropolitan area (see Fig. 3). It is run with the following options: 1.5-order closure prognostic-TKE-based GSPBL scheme (Shafran et al. 2000), force-restore soil model (Zhang and Anthes 1982), Rapid-Radiative Transfer Model (Mlawer et al. 1997) for longwave radiation, Dudhia shortwave radiation (Dudhia 1989), mixed-phase microphysics (Reis- 


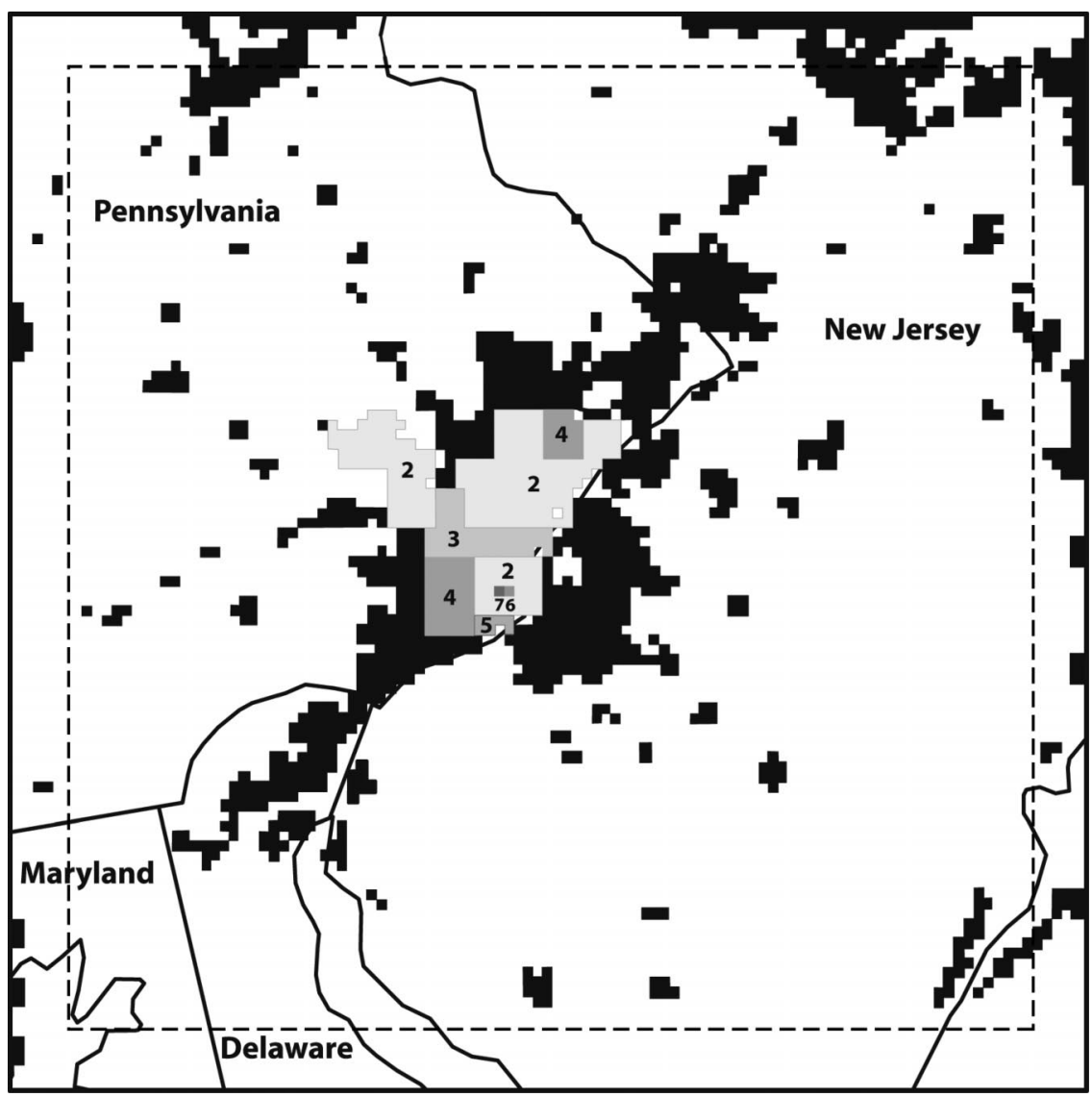

FIG. 3. The 1.33-km domain centered on Philadelphia. Grid cells designated as urban areas (by land use) are shaded. Blackened grid cells represent urban subcategory 1 . The remainder of the urban area is partitioned into urban subcategories $2-7$ as shown on the map. Refer to Tables 1 and 2 for definitions of these subcategories. The dashed box indicates the area shown in Fig. 4 .

ner et al. 1998), and explicit convection. FDDA is not used in the $1.33-\mathrm{km}$ domain so as to enable us to evaluate independently the influence of the UCP. The simulations with the $1.33-\mathrm{km}$ domain are initialized at 0000 UTC 14 July 1995 with initial and lateral boundary conditions interpolated from the simulation on the 4$\mathrm{km}$ domain. All simulations with the $1.33-\mathrm{km}$ domain cover 24-h periods ending at 0000 UTC 15 July 1995. To include the influence of urban obstacles, the UCP is used on the $1.33-\mathrm{km}$ domain with 40 layers that include 10 new layers in the lowest $100 \mathrm{~m}$ (lowest-layer depth of $4 \mathrm{~m}$ ). There are approximately 8 layers of the 40layer structure within the lowest layer of the 30-layer configuration, and 12 layers within the lowest $100 \mathrm{~m}$. Above $150 \mathrm{~m} \mathrm{AGL}$, the 30- and 40-layer structures are identical.

The GSPBL in MM5v3.5 computes the TKE and the vertical turbulent fluxes from the local gradients. Zhang et al. (2001) note that the GSPBL tends to underestimate the turbulent mixing and the mixing height. Bélair et al. (1999) also suggest that local closure schemes (like the GSPBL) tend to underestimate mixing inside the PBL during convective conditions. To improve the de- termination of the turbulent mixing in the GSPBL, the parameterization of the turbulent length scale of Bougeault and Lacarrère (1989), including a nonlocal feature in the turbulent diffusivity coefficient parameterization, is implemented in the GSPBL only for convective (unstable) conditions. The new mixing length in the GSPBL is derived from the potential upward and downward displacements that could be achieved by parcels with kinetic energy equal to the mean TKE before being stopped by buoyancy effects. This change increases the mixing, which also minimizes the tendency to generate nonphysical undulations in the $1.33-\mathrm{km}$ mixing height, wind, and temperature fields. These modifications are used for all simulations that use the GSPBL (i.e., the 4-km domain and all $1.33-\mathrm{km}$ simulations with and without the UCP).

The 1.33-km simulations use land-use data from the 30-s U.S. Geological Survey (USGS) 24-category database that accompanies MM5v3.5. In addition to the dominant land use category representation, the input to MM5 is modified to include the fractional percentages within each grid cell of each of the 24 land-use categories for use with the UCP. In the USGS 24-category 
TABLE 1. Morphology classifications used for UCP applications for Philadelphia. Urban subcategories in the left column were used in MM5 simulations on 1.33-km domain. Ellefsen (1991) urban terrain zones use the following abbreviations: A = attached buildings, Dc = detached close-set buildings, and Do = detached open-set buildings. Note that urban terrain zone Dc8 was not part of an urban subcategory, because there were no areas of Philadelphia classified by that urban terrain zone in Ellefsen (1991).

\begin{tabular}{cl}
\hline \hline Urban subcategory & \multicolumn{1}{c}{ Ellefsen (1991) urban terrain zones } \\
\hline 0 & None. Nonurban areas \\
1 & None. Urban areas (by land use classification) not included in Ellefsen's study \\
& A5: Commercial ribbon development, low to medium rise \\
& Dc3: Residential houses \\
& Dc5: Older commercial ribbons, less than five stories \\
& Do2: Residential apartments and row houses, low to medium rise \\
& A3: Apartments and abutted-wall houses, less than four stories \\
& A4: Industrial/storage, medium rise \\
& Dc2: Residential apartments and row houses \\
& Dc4: Industrial/storage, low rise \\
& Do1: Shopping centers, low rise \\
& Do3: Houses, low rise \\
& Do4: Truck-related industrial/storage, low rise \\
& Do5: Modern commercial ribbon development, low rise \\
& Do6: Administrative and cultural, low to medium rise \\
& A1: Commercial offices and retail, low to high rise \\
& A2: Apartments and hotels, four or more stories high \\
& Dc1: Commercial offices, high rise \\
\hline
\end{tabular}

classification, all urban areas are represented by a single category. The soil moisture for all $1.33-\mathrm{km}$ simulations is set from the MM5 summer climatological values as a function of dominant land use category (Grell et al. 1994). In simulations that use the roughness approach, the urban areas are assumed to be homogeneous (based on land use) and are characterized with the same physical attributes such as roughness length $z_{0}$, moisture availability, and albedo (Grell et al. 1994), which are also defined based on dominant land use category. For simulations with the roughness approach in the 1.33$\mathrm{km}$ domain, $z_{0}$ is set to $1.0 \mathrm{~m}$ for all urban areas.

\section{b. UCP input}

The UCP requires morphological parameters (e.g., $h_{c}$ and $\lambda_{f}$ ) that can be extracted from digital imagery (e.g., Ratti et al. 2002; Burian et al. 2002) and are commercially available from several vendors for various cities and with different degrees of accuracy and precision. We did not have access to a true urban morphology database for our area of interest, and so, for our UCP application, we added an urban subcategory overlay to the MM5 land use classification to account for some urban heterogeneity and to apply morphological characteristics. In an ideal situation, a true (commercially available) morphology database with variations from grid point to grid point would be used with the UCP. The characterization of the urban morphology here loosely follows Ellefsen (1991) who defines 17 urban terrain zones that represent age, intended use, construction, and height and density of buildings within the urban area. In this study, Ellefsen's urban terrain zones are aggregated to six categories [modified based on Grimmond and Oke (1999b)] to represent urban categories such as high rise, industrial, and urban residential (Table 1). A seventh category is added to cover urban areas within the modeling domain but outside of Ellefsen's study, and that category is generally assumed to be a lower-density urban area (e.g., suburban). The digital representation of the morphology of Philadelphia is adapted from a hand-drawn map of Philadelphia obtained from Ellefsen. Figure 3 shows the distribution of urban subcategories in the $1.33-\mathrm{km}$ computational domain. Approximately $15 \%$ of the grid cells in the computational domain are represented by urban area, and only about $20 \%$ of the urban grid cells have an urban subcategory that is derived from Ellefsen's study.

Each of the seven urban subcategories (Table 2) is characterized by a maximum building height $\left(h_{c}\right)$, ratio of the plan area of buildings to the surface area $\left(\lambda_{p}\right)$, and canyon fraction within each urban area $\left(f_{\text {cnyn }}\right)$ defined following Ellefsen (1991). The roof fraction $\left(f_{\text {roof }}\right)$ is deduced from $f_{\text {cnyn }}$. In the absence of specific data,

TABLE 2. UCP parameters associated with each urban subcategory.

\begin{tabular}{|c|c|c|c|c|c|c|c|}
\hline Urban subcategory & 1 & 2 & 3 & 4 & 5 & 6 & 7 \\
\hline Percentage of total urban area & 80.74 & 12.91 & 2.69 & 3.17 & 0.38 & 0.05 & 0.05 \\
\hline Lambda-P and lambda-F $\left(\lambda_{p}\right.$ and $\left.\lambda_{f}\right)$ & 0.15 & 0.30 & 0.40 & 0.10 & 0.15 & 0.60 & 0.15 \\
\hline Average building height $h_{c}(\mathrm{~m})$ & 3.0 & 10.0 & 11.0 & 6.0 & 10.0 & 45.0 & 90.0 \\
\hline Canyon fraction $f_{\text {cnyn }}$ & 0.85 & 0.70 & 0.60 & 0.90 & 0.85 & 0.40 & 0.85 \\
\hline Max anthropogenic heat flux $Q_{A \max }\left(\mathrm{W} \mathrm{m}^{-2}\right)$ & 50.0 & 100.0 & 100.0 & 100.0 & 100.0 & 100.0 & 100.0 \\
\hline
\end{tabular}


we assume that $A_{f}(z)$ and $A_{p}(z)$ are equivalent, and so the ratio of the frontal area of buildings to the surface area $\left(\lambda_{f}\right)$ is equivalent to $\lambda_{p}$, which may not always be a realistic assumption. For grid cells for which $f_{\text {urb }}$ is 1 , the sum of $\lambda_{p}$ and $f_{\text {cnyn }}$ is 1 . Ellefsen (1991) documented the average height of the buildings in each urban terrain zone for Philadelphia. Thus, we assumed for this study that all buildings in each grid cell are at the same (average) height, and so there is no variation of building height within a grid cell. We specifically define $A_{p}(z)$ $=\lambda_{p} / h_{c}$ for each urban subcategory.

For the UCP, $z_{0}$ is decreased considerably because the impact of the buildings (formerly roughness elements) on the flow is treated explicitly by the UCP. The $z_{0}$ now represents the roughness of the true surface (e.g., grass or pavement), which is commonly given by values between 0.01 and $0.10 \mathrm{~m}$; we set $z_{0}$ to $0.05 \mathrm{~m}$ for all urban subcategories.

Because roofs in the Philadelphia area are largely composed of brick, concrete, and tarpaper shingles, we set $\alpha_{\text {roof }}$ to 0.12 and $\varepsilon_{\text {roof }}$ to 0.90 in Eq. (7), values which are consistent with values for those surfaces as defined by the American Society of Heating, Refrigerating, and AirConditioning Engineers (ASHRAE 1967). For the hot summer day in our study, $Q_{\text {Amax }}\left[\right.$ Eq. (8)] is $50 \mathrm{~W} \mathrm{~m}^{-2}$ for suburban areas (see Table 2). From Fig. 2, this leads to peak values near $47 \mathrm{~W} \mathrm{~m}^{-2}$ in daytime and nocturnal values near $10 \mathrm{~W} \mathrm{~m}^{-2}$, which is reasonably consistent with Grimmond and Oke (1995) and Sailor and Fan (2002). For all other urban areas, including the urban core, $Q_{A \max }$ is set to $100 \mathrm{~W} \mathrm{~m} \mathrm{~m}^{-2}$. This leads to peak daytime values near $95 \mathrm{~W} \mathrm{~m}^{-2}$ and nocturnal values near $20 \mathrm{~W} \mathrm{~m}^{-2}$, which more closely (and somewhat conservatively) follow the potential peak values suggested by Masson (2000).

\section{Results}

This study focuses on simulating the meteorological fields at finescales (i.e., 1.33-km horizontal grid spacing) for Philadelphia on 14 July 1995, a day characterized by clear skies and significant daytime heating. Winds in the Philadelphia area were generally southwesterly, and maximum temperatures of $35^{\circ}-40^{\circ} \mathrm{C}$ were observed throughout the region. This case was studied for its airquality characteristics under the field program of the North American Research Strategy for Tropospheric Ozone in the northeastern United States (NARSTO$\mathrm{NE}$ ). Additional discussion of the meteorological and chemical behavior of this case can be found in the literature (Berman et al. 1999; Seaman and Michelson 2000).

Several simulations are made with the $1.33-\mathrm{km}$ domain to determine the impact of the UCP on the MM5 simulation. First, the 30-layer simulation (hereinafter: nocan30) represents a "standard" application of MM5 with the roughness approach. Second, the 40-layer simulation with the UCP (hereinafter: can40) demonstrates the influence of the drag-force approach with the simplified urban thermodynamics. Third, the 40-layer simulation with the roughness approach (hereinafter: nocan40) is an intermediate case that is used to assess whether the changes between nocan 30 and can 40 are related to the change in vertical structure. We note that the use of the Monin-Obukhov similarity theory may be less applicable because of the fine vertical resolution near the ground in our 40-layer configuration, and so nocan40 is not considered to be a realistic application of MM5. Comparisons are made among nocan30, can40, and nocan40 on fields above the urban canopy, on vertical profiles located at the city center, and with meteorological observations. These comparisons show the impact of the UCP on the turbulent structures of the URSL (where pollutants are emitted) and on the PBL height (the effective volume in which the pollutants are dispersed). Last, a brief discussion of the sensitivity of the simulations with the UCP to the dynamic and thermodynamic effects is presented.

\section{a. Fields above the urban canopy}

Fields are examined just above the urban canopy to show differences in the development of fields in the URSL. At this level, the meteorological fields are strongly influenced by the heat and momentum fluxes from the canopy below. Simulation of the fields at this level is critical because pollutants are transported from the canopy to neighboring areas via this level. Figure 4 is a comparison of the roughness approach in nocan 30 with the UCP in can40. Figure $4 \mathrm{a}$ is the temperature and wind vectors at $55 \mathrm{~m} \mathrm{AGL}$ (generally above the urban canopy) at 2000 UTC [1600 local daylight time (LDT)] for can40. A warmer region is centered on the urban core and downwind of the city. The winds are generally southwesterly at 3-5 $\mathrm{m} \mathrm{s}^{-1}$, with some convergence over the urban area because of the concentrated heat over the city. Figure $4 \mathrm{~b}$ is the difference in temperature and wind vectors for can40 (shown in Fig. 4a) minus nocan30. A large area in and downstream of the urban core is about $1 \mathrm{~K}$ warmer in can 40 than in nocan30. The explicit treatment of the sources of urban heating with the UCP contributes to a larger temperature gradient between the urban and rural zone in can 40 than in nocan30, and, thus, a stronger urban heat island effect in can40. The wind vector differences in the urban core in Fig. 4b are northerly or northeasterly, which reflects a reduction in the overall southwesterly flow in can 40 of up to $1 \mathrm{~m} \mathrm{~s}^{-1}$ in some places from the drag captured by the UCP. This result suggests that the reduction of winds due to drag from the representation of urban structures in can40 leads to drag in the URSL above the canopy, as well. Differences in temperature and wind speed outside the urban core are generally small.

In the Gayno-Seaman PBL scheme in MM5, PBL height is determined from the TKE profile, and it is the height at which the TKE drops below a threshold value. 
a)

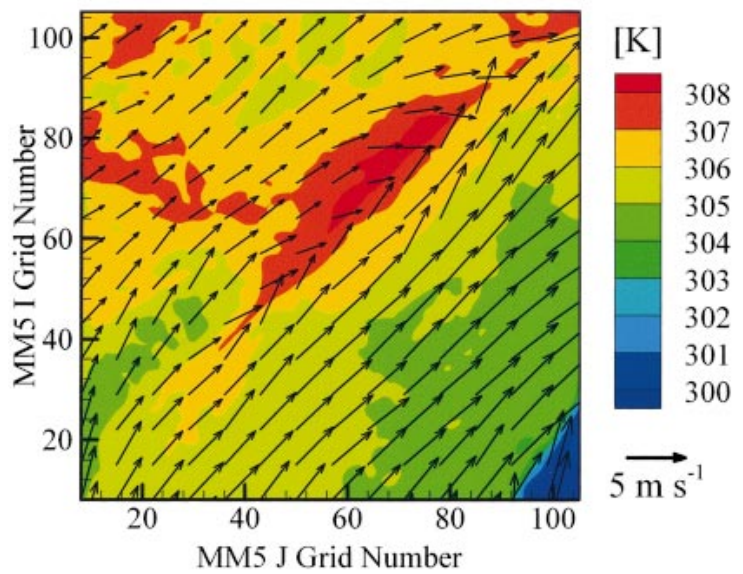

c)
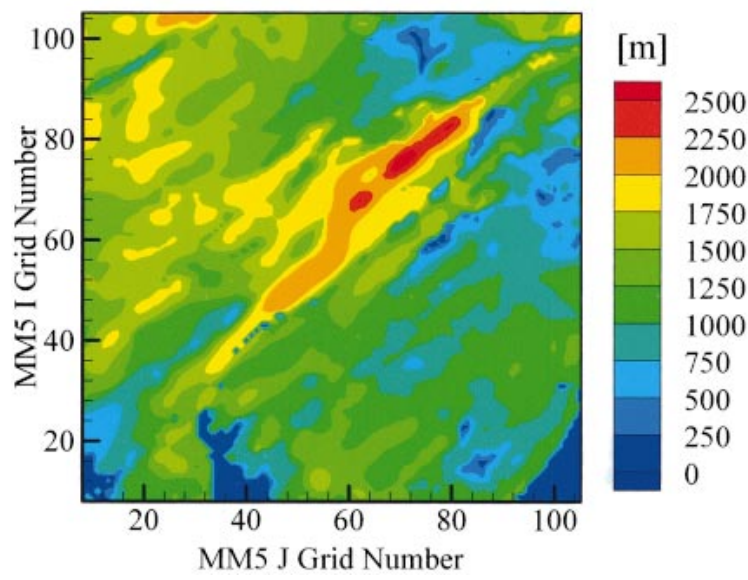

b)

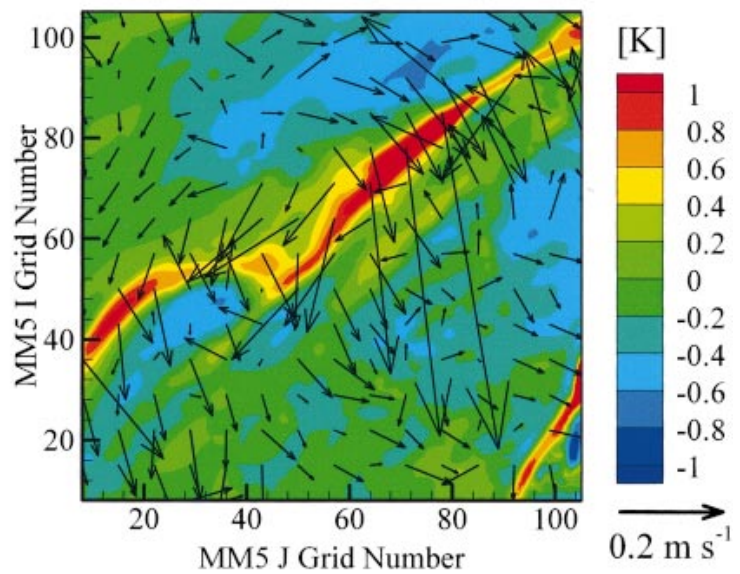

d)

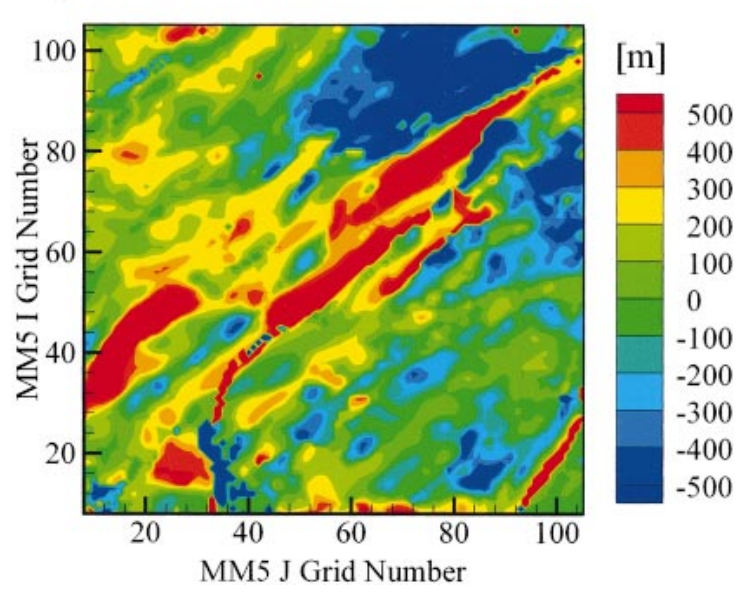

FIG. 4. Horizontal variations in meteorological fields valid at 2000 UTC 14 Jul 1995. Values along the $x$ and $y$ axes are gridpoint counters. Refer to Fig. 3 for geography of the area shown here. (a) Temperature (K) and wind vectors (m s${ }^{-1}$ ) at 55 m AGL for can40. (b) Temperature difference $(\mathrm{K})$ and wind vector difference $\left(\mathrm{m} \mathrm{s}^{-1}\right.$ ) at $55 \mathrm{~m} \mathrm{AGL}$ : can40 minus nocan30. (c) PBL height (m) from can40. (d) PBL height difference $(\mathrm{m})$ : can40 minus nocan 30 .

Figure 4c shows the PBL height calculated in MM5 at 2000 UTC (1600 LDT), or approximately the maximum mixing height, for can40. The PBL is well mixed, and a large area of the urban core contains PBL heights above $2000 \mathrm{~m}$ that are advected downwind of the city. (The areas of low PBL heights along the southern boundary of the domain are associated with water bodies.) Figure $4 d$ is the PBL height difference for can 40 (shown in Fig. 4c) minus nocan30. The PBL heights in the urban core with can40 are generally 300-500 m higher than with nocan30. This result can be explained by the higher air temperature in can40, which is induced by the thermodynamic component of the UCP. In addition, the UCP in can40 explicitly enhances the TKE in the urban canopy, in particular at the rooftop level, which results in additional mixing in the URSL and a deeper PBL than that from the roughness approach used in nocan30. At this time, outside the urban area, the differences in PBL height between can40 and nocan30 are generally less pronounced. Differences in the PBL heights of less than $50 \mathrm{~m}$ in nonurban areas are generally observed near local noon (not shown), as are increases in PBL height of $150 \mathrm{~m}$ or more in the urban core with the UCP during nighttime (not shown).

Figure 5 shows the evolution of the PBL height at the city center (urban subcategory 6 in Fig. 3). As in Fig. 4, the PBL height during the afternoon period is consistently lower for the cases using the roughness approach. Both of those cases reach their peak values of PBL height between 2100 and 2200 UTC (1700 and 


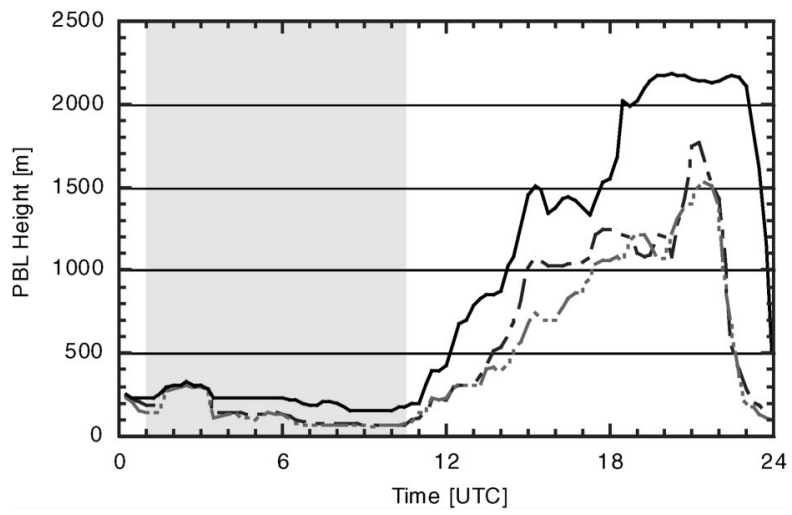

FIG. 5. Time series of PBL height $(\mathrm{m})$ at the city center (subcategory 6 in Fig. 3) valid 0000 UTC 14 Jul-0000 UTC 15 Jul 1995 for nocan30, nocan40, and can40. Nighttime hours are shaded. Dashdot lines represent nocan30, dash-triple dot lines represent nocan40, and solid lines represent can 40 .

1800 LDT), with maxima near 1800 and $1500 \mathrm{~m}$ for nocan30 and nocan40, respectively. In those cases, the PBL rapidly collapses shortly after reaching the peak value. By contrast, the PBL grows much deeper and more rapidly with the UCP in can40. The PBL height in can40 exceeds $2000 \mathrm{~m}$ by 1800 UTC (1400 LDT) and reaches its peak plateau near 1900 UTC (1500 LDT) of between 2100 and $2200 \mathrm{~m}$, which is sustained until just before 2300 UTC (1900 LDT). In can40, the heat released within the urban canopy contributes to sustaining a deeper PBL than in nocan30 and nocan 40. Berman et al. (1999) show that the maximum PBL height (estimated from meteorological observations) in the Philadelphia urban core for 14 July 1995 is near 2100 or $2200 \mathrm{~m}$, which is consistent with the simulation of can40. In addition, Berman et al. (1999) show that the maximum PBL height in 13 ozone-episode days (including 14 July) in the summer of 1995 is greater than $2000 \mathrm{~m}$ in the Philadelphia urban core. The analysis by Berman et al. (1999) also shows that the maximum PBL depth in the Philadelphia area is typically reached near 1400 eastern standard time (EST; 1500 LDT) for that 13-day period, and it drops to about $1750 \mathrm{~m}$ by 1800 EST (1900 LDT). The UCP simulation in can40 very closely follows the PBL evolution reported by Berman et al. (1999) for 14 July (and other similar days in 1995), and it is clearly superior to the mixing depths predicted by nocan 30 and nocan 40 . Thus, the increase in air temperature induced by the thermodynamic component of the UCP works to increase the PBL height, especially in the late afternoon and overnight. From this result, we could expect a large impact on pollutant concentrations because of the modification of the effective volume over which pollutants are dispersed.

\section{b. Vertical profiles within urban canopy}

In the absence of measured vertical profiles for Philadelphia for the day of interest, here we examine nor- malized model profiles from our study to identify whether the UCP is successful at capturing the structure in and above the URSL. Figure 6 shows vertical profiles at the same point used for Fig. 5 (urban subcategory 6 in Fig. 3). Figure 6a is the 1200 LDT local friction velocity $u_{*}$ normalized by its maximum in can40 and plotted against normalized height $z / h$, where $h$ is the average building height (in this case $h_{c}$ from our UCP formulation, which is $45 \mathrm{~m}$ for this cell). Martilli et al. (2002), using normalized $u_{*}$ data from Rotach (2001) based on a combination of measurements and wind tunnel studies, show that $u_{*}$ is maximized at $z / h \approx 2$ (near the top of the canopy in that study) and that there is a sharp decrease of $u_{*}$ toward the ground (i.e., a rapid increase with height). For the nocan30 and nocan 40 cases, the normalized $u_{*}$ profile is generally constant in the URSL, which is consistent with the constant-fluxlayer theory used by the roughness approach. In nocan30, the buildings are mostly below the lowest model layer, and so there is clearly no structure within the (unresolved) urban canopy. Although there are layers within the urban canopy in nocan40, the profile is nearly constant in the lowest $100 \mathrm{~m}$. In can 40 , the profile shape matches reasonably well with measurements given by Rotach (2001) and model results achieved by Martilli et al. (2002) for an idealized case. In can40, the maximum value of $u_{*}$ is achieved near $z / h=1$ (top of the canopy in this study); the value of the maximum is somewhat lower than those shown by measurements and other modeling studies. In our configuration, however, all buildings have the same height, whereas in Martilli et al. (2002) the urban canopy layer extends to $z / h \approx$ 2.4 (or approximately where the $u_{*}$ profile is maximized in that study). Nevertheless, the use of the UCP in can40 generates profiles of $u_{*}$ that maintain the same shape for urban areas as measurements and wind tunnel studies shown in Rotach (2001), whereas the roughness approach used in nocan 30 and nocan 40 does not.

Figure $6 \mathrm{~b}$ is the ratio between TKE and the square of the maximum value of the local $u_{*}$ at 1200 LDT plotted against $z / h$ (as in Fig. 6a). Both nocan 30 and nocan 40 have TKE profiles that slightly decrease toward the ground because of the influence of the lower boundary conditions. When the UCP is used in can40, the TKE profile increases rapidly with height, with a subtle maximum near the mean level of the building roofs $(z / h \approx$ 1). Monthly measurements by Christen et al. (2002) and wind tunnel measurements by Kastner-Klein et al. (2001) show that TKE is maximized at $z / h \approx 1$. In addition, the nonnormalized urban TKE profiles for midday (not shown) have a sharp maximum of $5-10 \mathrm{~m}^{2} \mathrm{~s}^{-2}$ at the rooftop level for can 40 , while nocan 30 and nocan 40 have subtle maxima of $1-1.5 \mathrm{~m}^{2} \mathrm{~s}^{-2}$ at a height of nearly $100 \mathrm{~m}$ above the rooftop level. The TKE maximum is larger at night (Fig. $6 \mathrm{c}$ ) because of the decreased mixing in the PBL overnight. $\mathrm{Vu}$ et al. (2002) and Martilli et al. (2002) also simulated this shape for TKE profiles in stable atmospheres. Overall, the TKE profiles generated by the UCP 


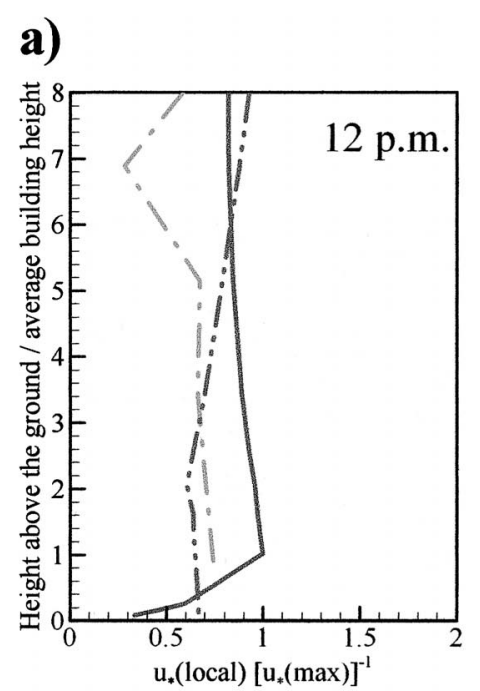

b)

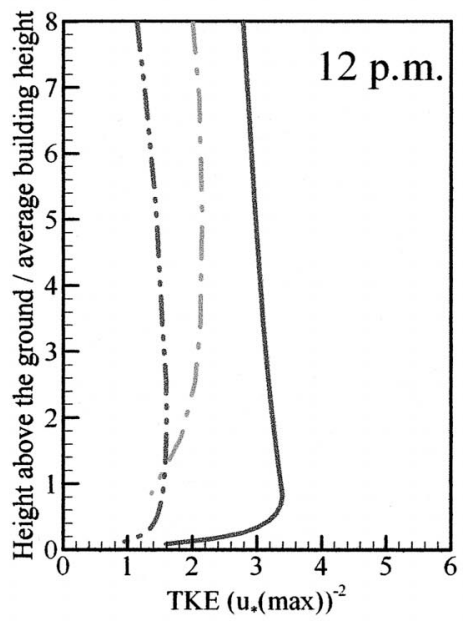

e)

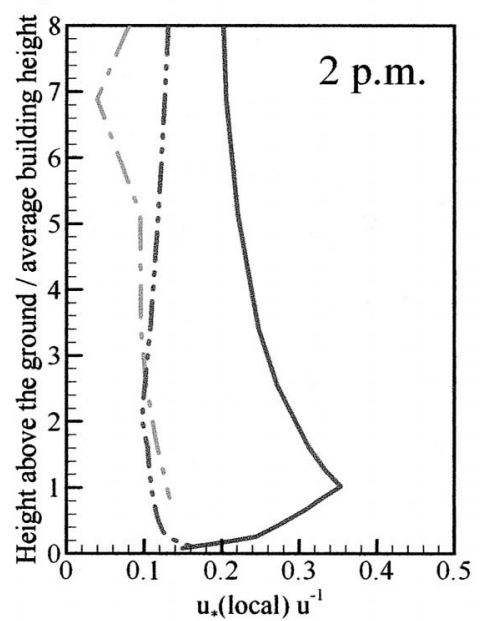

c)

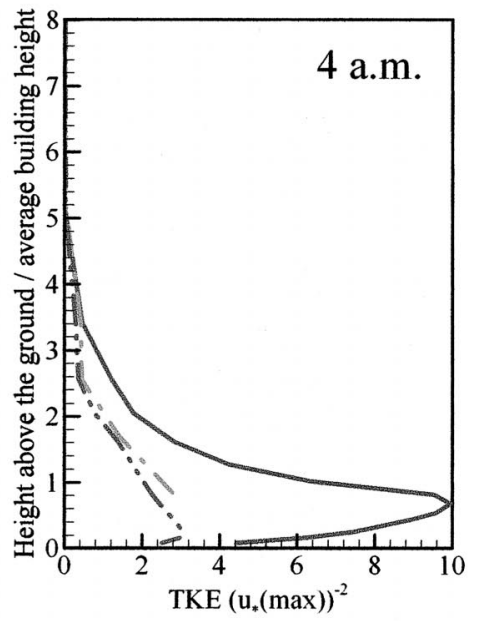

f)

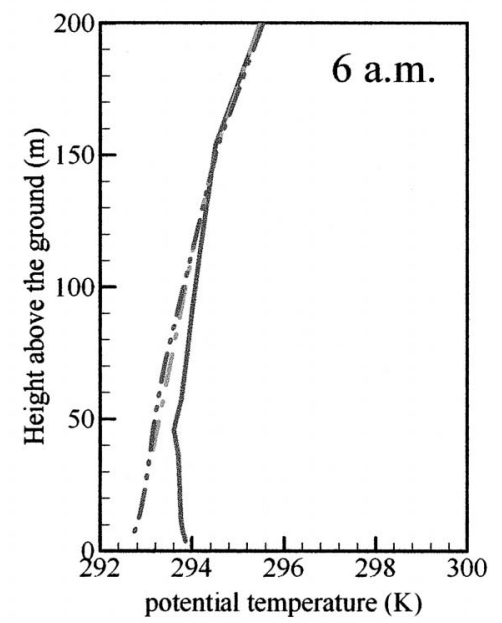

FIG. 6. Vertical profiles taken at the city center (urban subcategory 6 in Fig. 3). Dash-dot lines represent nocan30, dash-double dot lines represent nocan40, and solid lines represent can40. (a) The local $u_{*}$ normalized by its maximum value at 1200 LDT. (b) The ratio between TKE and the square of the maximum value of the local $u_{*}$ at 1200 LDT. (c) Same as in (b), but for 0400 LDT. (d) Wind speed at 1400 LDT. (e) The local $u_{*}$ normalized by wind speed at 1400 LDT. (f) The air potential temperature at 0600 LDT.

are more consistent with observed profiles than are those generated by the roughness approach for urban areas.

Figure 6d shows the mean wind speed at 1400 LDT. As expected, the winds are greatly reduced in can 40 when compared with nocan 30 and nocan 40 . The relative reduction of wind speed in can40 is seen throughout the URSL, which is consistent with the wind speed reduction above the canopy in Fig. 4. Figure 6e shows $u_{*}$ normalized by the mean wind speed at the same time as in Fig. 6d. Both nocan30 and nocan40 have nearly constant profiles in the urban canopy. By contrast, can40 shows some structure that reflects a maximum value at the rooftop level. This relation accounts for both the reduction of wind speed in the urban canopy with the UCP (Fig. 6d) and the maximized $u_{*}$ at the rooftop level (Fig. 6a).

Figure $6 \mathrm{f}$ is a profile of air potential temperature at
0600 LDT plotted against height above ground level (not normalized, and on a different vertical scale than that used in Figs. 6a-e). Both nocan30 and nocan 40 have clearly stable profiles through the lowest $200 \mathrm{~m}$. The profile of potential temperature for can40 is somewhat neutral through the lowest $150 \mathrm{~m}$, with an unstable layer through the depth of the urban canopy $(45 \mathrm{~m}$ at this cell), which is consistent with the reduction of the atmospheric stability observed near urban surfaces (Roth 2000). In addition, the depth of the neutral layer in can40 is in agreement with the values given by Oke (1995), who notes that the urban boundary layer often exhibits a neutral or slightly stable layer that extends up to heights of 100-300 m. The air potential temperature near the surface in can 40 is about $1.5^{\circ} \mathrm{C}$ warmer than in nocan 30 and nocan40, which illustrates the nocturnal urban heat island. 


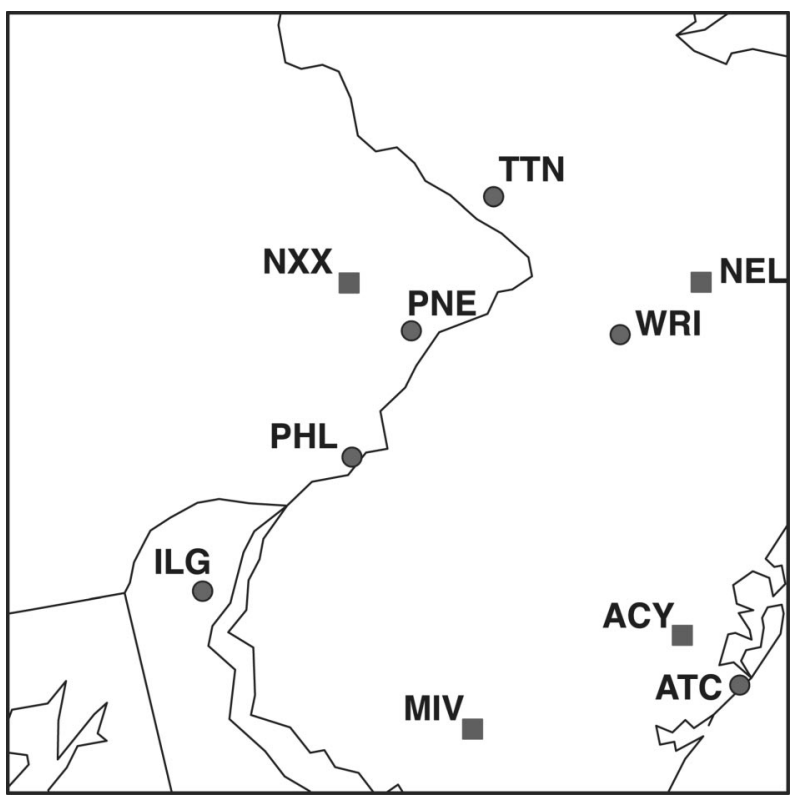

FIG. 7. Observation sites used to calculate statistical measures. Stations corresponding to urban grid cells are denoted by circles; nonurban (i.e., rural) sites are denoted by squares. All of the stations are standard NWS observing stations with the exception of ATC, which is a special NARSTO-NE site.

The vertical profiles at a rural site (not shown) indicate that there is virtually no impact on the fields from using the UCP, as expected. Thus, the UCP in can40 can generate profiles of wind speed, $u_{*}$, TKE, and potential temperature in the URSL that are consistent with available measurements without disrupting the vertical profiles in the rural areas. The cases with the roughness approach generally show little distinction between urban and rural boundary layer structures at this scale. Overall, there are important differences between the model results using the UCP and the roughness length approaches, and measurements from other urban field data and wind tunnel experiments suggest that the UCP improves model results in urban areas.

\section{c. Comparison with surface observations}

Comparisons with meteorological data are made for nine U.S. National Weather Service (NWS) surface observation sites and surface data from one special sounding from the NARSTO-NE field experiment (Fig. 7) to illustrate the spatial impact of the UCP in our modeling domain over areas where the UCP is specifically applied (i.e., urban areas) as well as in areas where the parameterization is benign (i.e., nonurban or "rural" areas). We recognize that these single-station measurements cannot capture the spatial variability within the MM5 grid cells and that heterogeneity can be exacerbated in urban areas at this horizontal scale. In an ideal situation, a high sampling density of measurements would be used to provide a spatial average to validate the performance of the model. For this evaluation, the surface temperature measurement height is $2 \mathrm{~m} \mathrm{AGL}$, and the wind measurement height is $10 \mathrm{~m}$ AGL. The prognostic fields from the 30-layer nocan30 case are reduced from the lowest model midlayer ( $\sim 19 \mathrm{~m}$ AGL) to the observation height by using the similarity-theory relations from the GSPBL. The fields from the 40-layer simulations (nocan40 and can40) are taken from the closest model midlayer to the measurement height (less than 1-m difference for both variables). Not all of the observation sites reported data for each meteorological variable at each hour.

Figure 8 is a set of time series of temperature for the three cases compared with observations for two urban sites and one rural site. Figure 8 a shows the temperature evolution at urban site Wilmington, Delaware (ILG in Fig. 7). Both nocan 30 and nocan 40 have nearly the same temperature pattern overnight. The nighttime temperatures for can40 represent an improvement at each hour of $1^{\circ}-1.5^{\circ} \mathrm{C}$, although an obvious cold bias still exists. The improvements in can40 are related to the sources of energy in urban areas that are not considered with the roughness approach. Note that the initial temperature fields are consistently $1^{\circ}-2^{\circ} \mathrm{C}$ colder than observations (Fig. 8) and that can40 nearly overcomes this handicap when the minimum temperature is reached at ILG. When the daytime heating occurs, the temperature rise in nocan30 is far more pronounced than in nocan 40 and can40, and this fact is likely related to the change in vertical resolution in those cases. The 40-layer cases tend to match better with the observations than does nocan30. The cases that use the roughness approach converge to the same (sinking) temperature trace by 2300 UTC (perhaps related to the early PBL collapse in those simulations), whereas can40 tends to hold the urban heat longer following the observations. The improvements in can40 can be attributed to the mechanisms in the UCP that include the trapping of radiation in canyons and the contributions to the energy budget from anthropogenic sources, which are not handled with the roughness approach.

The cases show a similar pattern as for ILG for the urban site, Philadelphia Northeast Airport (PNE in Fig. 7), that is downwind of the urban core (Fig. 8b). Like at ILG, the nighttime temperature patterns for nocan 30 and nocan 40 are nearly identical, and they are $4^{\circ}-6^{\circ} \mathrm{C}$ colder than the observations. Case can40, although still colder than observations, reduces the cold bias at PNE during nighttime hours by about $2^{\circ} \mathrm{C}$. During daytime, the simulated temperatures in can 40 tend to be in phase and to match better with observations than do those in nocan 30 and nocan 40 . In nocan 30 , the maximum temperature is within about $0.5^{\circ} \mathrm{C}$ of observations, but it is reached approximately $4 \mathrm{~h}$ too early. In nocan 40 , the maximum temperature is about $2^{\circ} \mathrm{C}$ too cold, and it is reached about $2 \mathrm{~h}$ too early. It is likely that the can 40 temperature traces are more realistic for the urban sites as a result of the improvements in simulating the urban 
a)

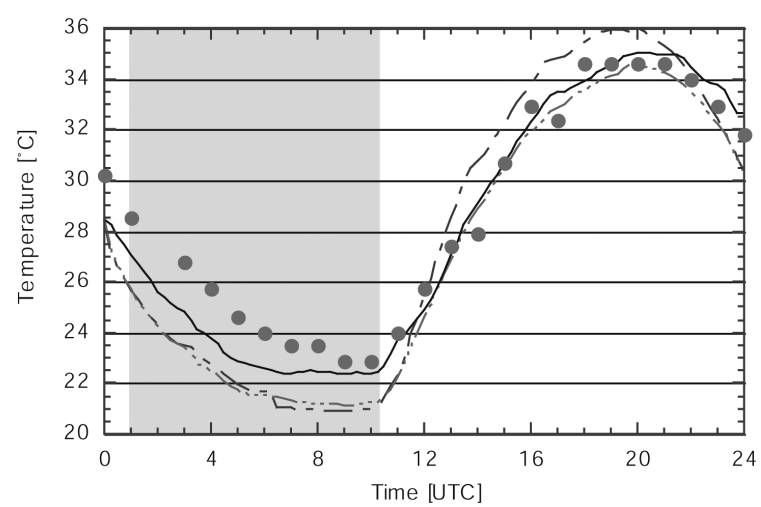

b)

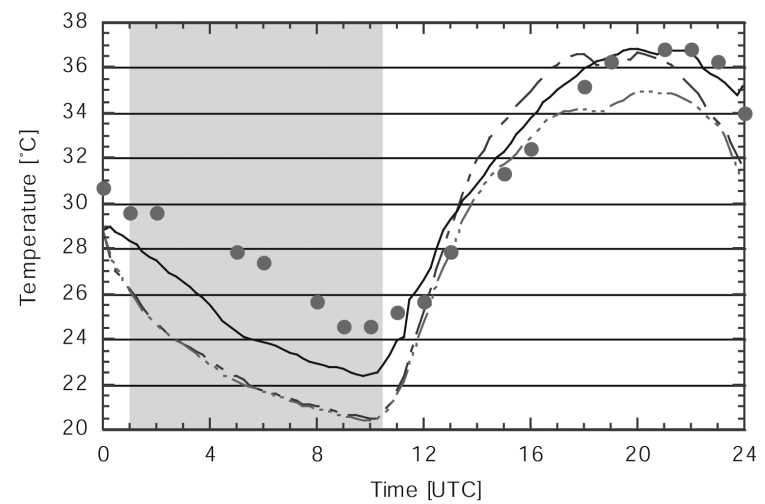

c)

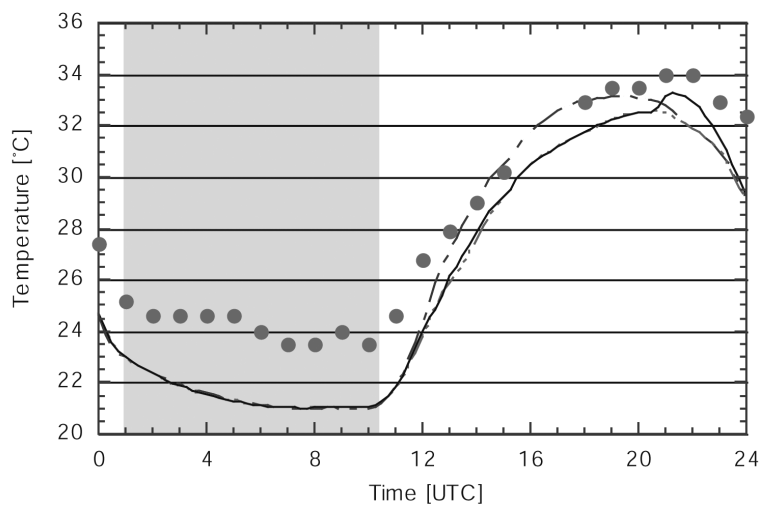

FIG. 8. Time series of temperature $\left({ }^{\circ} \mathrm{C}\right)$ at three observation sites, valid 0000 UTC 14 Jul-0000 UTC 15 Jul 1995 for nocan30, nocan40, and can40. Nighttime hours are shaded. Large filled circles represent observations, dash-dot lines represent nocan30, dash-triple dot lines represent nocan40, and solid lines represent can40. (a) Urban point at ILG. (b) Urban point at PNE. (c) Nonurban (i.e., rural) point at MIV.

boundary layer using the drag-force approach with the simplified thermodynamics modifications.

Figure $8 \mathrm{c}$ shows the temperature time series for a nonurban (i.e., rural) site, Millville, New Jersey (MIV in Fig. 7). At MIV, all three cases have very similar overnight temperatures, as expected. The UCP should have no direct influence on nonurban sites far upstream of the city, such as MIV. In addition, the same nocturnal cold bias is seen at MIV as in the roughness-approach simulations for ILG. As the daytime heating occurs, nocan30 has the same rapid temperature rise at MIV as at ILG, where the two 40-layer simulations maintain nearly the same time series. The nocan 30 case reaches its peak temperature $2-3 \mathrm{~h}$ earlier than observations, and can40 reaches the peak in a similar phase to the observations. This change at MIV in can40 may be related to advection from a nearby cluster of urban grid cells that were treated with the UCP. Although can40 is generally similar to nocan40, as expected at MIV, can40 is more skillful for the maximum temperature than is nocan40 despite the absence of direct forcing from the UCP at this nonurban site.

Figure 8 clearly shows a cold bias in the initial state for the $1.33-\mathrm{km}$ domains at all three sites. The temperature fields are initialized here by interpolating horizontally (and vertically, for the 40-layer simulations) from the simulation on the 4-km parent domain using the standard procedure in MM5 for a one-way-nesting application. One possible source of the poor initial state is the somewhat primitive treatment of the soil moisture and soil model in the version of MM5 that we used for this study. The soil moisture values used here are based on climatological values as a function of dominant land use category as given by the standard MM5 land use lookup tables. The soil model we use (slab) is the only soil model that is currently compatible with the GaynoSeaman PBL scheme. A recent focus of research within the MM5 community has been the development and use of more sophisticated land surface models (LSMs) that account for soil moisture based on precipitation patterns and more realistic input data. These LSMs have been shown to improve the simulation of the surface fluxes and thus the near-surface air temperature in MM5 (e.g., Xiu and Pleim 2001; Chen and Dudhia 2001). Another version of the model was run for multiple days and the same nocturnal cold bias persisted throughout the simulation in regions where the UCP was not used (not shown). Figure 8 (near hour 24) suggests that the nocturnal cold bias will remain for the simulations without the UCP and for the rural site in the simulation with the UCP. Figure 8 also suggests that the nocturnal temperatures at the urban sites might be improved on subsequent days by using the UCP.

Several statistical measures are also computed using the sites shown in Fig. 7. Willmott (1982) suggests a variety of measures to quantify model performance (see the appendix). Figure 9 shows time series of rmse for temperature and vector wind difference (VWD; see the appendix) calculated only for the six urban sites (see Fig. 7) for 14 July 1995. In Fig. 9a, the temperature rmse is consistently lower for can40 than for nocan 30 and nocan 40 throughout the night. The hourly rmse values in can40 actually drop below the initial error for several hours. As the PBL grows in the midmorning, the results are not as consistent. However, by 1800 UTC (1400 LDT), can40 is again the best of the three cases for all urban sites for the remainder of the day. Overall, 
a)

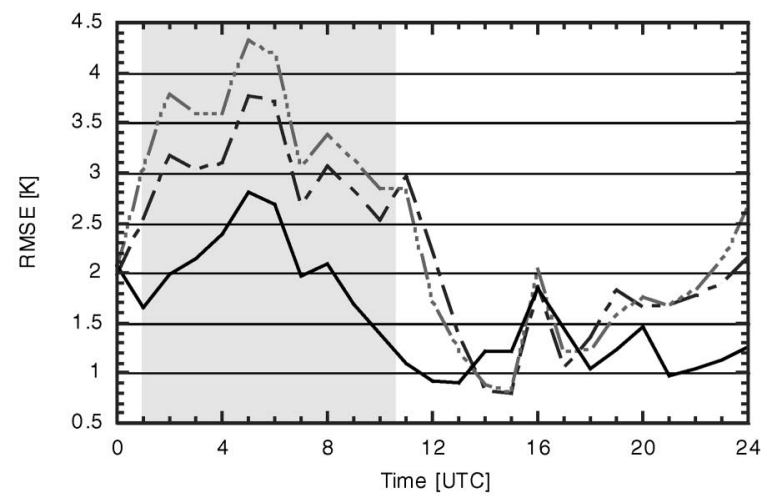

b)

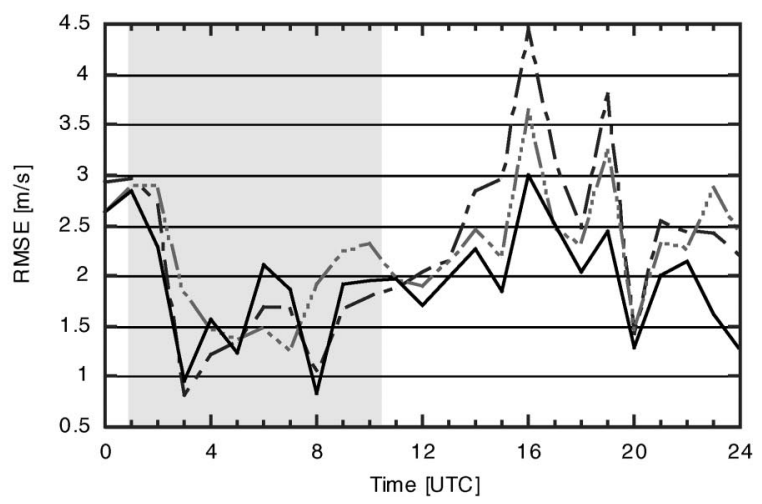

FIG. 9. Time series of rmse for (a) temperature and (b) VWD for urban sites (as shown in Fig. 7), valid 0000 UTC 14 Jul-0000 UTC 15 Jul 1995 for nocan30, nocan40, and can40. Nighttime hours are shaded. Dash-dot lines represent nocan30, dash-triple dot lines represent nocan40, and solid lines represent can 40 .

the rmse in can 40 is below $1.5^{\circ} \mathrm{C}$ for all but one of the daytime hours. The positive result in can40 can be attributed to the ability to simulate better the nocturnal urban heat island effects, as well as the improved maximum daytime temperatures, by specifically including urban radiation sources. For the VWD (Fig. 9b), there is no clear trend in verification overnight, because each case is best at various hours. However, during daytime hours, can40 is the best of the three cases at nearly every hour. In fact, can40 maintains an rmse of less than 2.0 $\mathrm{m} \mathrm{s}^{-1}$ at 15 of the $24 \mathrm{~h}$ and is less than $3.0 \mathrm{~m} \mathrm{~s}^{-1}$ at each hour throughout the day. When both fields are considered together, can40 outperforms the simulations of nocan30 and nocan 40 overall for the urban sites.

Table 3 contains the aggregate 24 -h statistics for all 10 stations (see Fig. 7) for 14 July 1995 (excluding initial time) for nocan30, nocan40, and can40. For temperature, can40 is consistently superior to nocan30 and nocan40 for all statistical categories. The mean absolute error (MAE) and rmse are reduced in can40 by $0.4-0.6$ $\mathrm{K}$, and the mean error (MEAN ERR) is reduced by $0.6-$
TABLE 3. Aggregate 24-h statistics for 14 Jul 1995 for all stations for three cases: nocan 30 , nocan 40 , and can40. Here, $T$ is temperature and WS is wind speed.

\begin{tabular}{|c|c|c|c|}
\hline Statistic & Nocan 30 & Nocan40 & Can40 \\
\hline$T: \operatorname{MAE}(\mathrm{K})$ & 2.00 & 2.21 & 1.62 \\
\hline$T: \operatorname{MEAN}$ ERR $(\mathrm{K})$ & -1.85 & -2.03 & -1.20 \\
\hline$T$ : IA (dimensionless) & 0.94 & 0.93 & 0.96 \\
\hline$T:$ Rmse $(\mathrm{K})$ & 2.26 & 2.51 & 1.87 \\
\hline$T:$ Rmses $(\mathrm{K})$ & 1.87 & 2.07 & 1.29 \\
\hline$T:$ Rmseu $(\mathrm{K})$ & 1.26 & 1.42 & 1.36 \\
\hline$T$ : SYS (fraction) & 0.69 & 0.68 & 0.48 \\
\hline$T$ : UNSYS (fraction) & 0.31 & 0.32 & 0.52 \\
\hline WS: $\operatorname{MAE}\left(\mathrm{m} \mathrm{s}^{-1}\right)$ & 1.29 & 1.18 & 0.99 \\
\hline WS: $\operatorname{MEAN} \operatorname{ERR}\left(\mathrm{m} \mathrm{s}^{-1}\right)$ & 1.07 & -0.47 & -0.13 \\
\hline WS: IA (dimensionless) & 0.55 & 0.57 & 0.62 \\
\hline WS: Rmse $\left(\mathrm{m} \mathrm{s}^{-1}\right)$ & 1.64 & 1.47 & 1.26 \\
\hline WS: Rmses $\left(\mathrm{m} \mathrm{s}^{-1}\right)$ & 1.34 & 0.90 & 0.77 \\
\hline WS: Rmseu $\left(\mathrm{m} \mathrm{s}^{-1}\right)$ & 0.96 & 1.16 & 1.00 \\
\hline WS: SYS (fraction) & 0.66 & 0.38 & 0.37 \\
\hline WS: UNSYS (fraction) & 0.34 & 0.62 & 0.63 \\
\hline
\end{tabular}

$0.8 \mathrm{~K}$. The MEAN ERR shows that a cold bias exists for all cases but is less pronounced in can40. The index of agreement (IA) is higher with can40, which suggests that it is a better model for temperature. The generally high IA for all cases results from the model's ability to capture the diurnal tempeature cycle. The improvements in the urban nocturnal temperatures (as shown in Fig. 8a) account for some of the statistical advantage with can40. However, 4 of the 10 observation sites represent nonurban areas, where there is little change in the nocturnal temperatures (Fig. 8b) and no direct influence from the UCP. The statistics indicate that improvements are made throughout the domain. The systematic error (SYS) and the unsystematic error (UNSYS) show that can40 greatly improves the proportion of errors as compared with nocan30 and nocan40. Both nocan30 and nocan40 have about two-thirds of the error attributable to systematic error, whereas it is less than one-half in can40.

Table 3 also shows the statistical measures for wind speed for the three cases. As with the temperature, can 40 is consistently superior to nocan30 and nocan40 in all statistical categories. The MEAN ERR for wind speed shows that nocan 30 tends to overpredict and nocan 40 tends to underpredict wind speed, whereas can40 slightly underpredicts wind speed. There is an improvement of $0.2-0.3 \mathrm{~m} \mathrm{~s}^{-1}$ in MAE with can40. The IA in can40 is higher, and the rmse is lower by $0.2-0.4 \mathrm{~m} \mathrm{~s}^{-1}$. SYS is much lower in both nocan40 and can40 than in nocan30, which also suggests that the vertical structure may have an influence. Although the absolute magnitude of the improvement is small with the UCP, note that the observed wind speeds were generally $2-4 \mathrm{~m} \mathrm{~s}^{-1}$ on 14 July, and so these changes could be important. In addition, statistics for wind direction (not shown) indicate that can 40 decreases the MEAN ERR by $4.5^{\circ}$ and rmse by $3^{\circ}$ when compared with nocan 30 and nocan 40 . These improvements in wind speed and direction in can 40 , 
TABLE 4. Same as in Table 3, but for can40, en_only, and mom_only.

\begin{tabular}{lrrr}
\hline \hline \multicolumn{1}{c}{ Statistic } & Can40 & En_only & Mom_only \\
\hline$T:$ MAE (K) & 1.62 & 1.71 & 2.04 \\
$T:$ MEAN ERR (K) & -1.20 & -0.83 & -1.91 \\
$T:$ IA (dimensionless) & 0.96 & 0.96 & 0.94 \\
$T:$ Rmse (K) & 1.87 & 2.00 & 2.32 \\
$T:$ Rmses (K) & 1.29 & 1.11 & 1.96 \\
$T:$ Rmseu (K) & 1.36 & 1.67 & 1.25 \\
$T:$ SYS (fraction) & 0.48 & 0.31 & 0.71 \\
$T:$ UNSYS (fraction) & 0.52 & 0.69 & 0.29 \\
WS: MAE (m s ${ }^{-1}$ ) & 0.99 & 1.29 & 1.06 \\
WS: MEAN ERR (m s $\left.{ }^{-1}\right)$ & -0.13 & -0.50 & -0.23 \\
WS: IA (dimensionless) & 0.62 & 0.55 & 0.61 \\
WS: Rmse (m s ${ }^{-1}$ ) & 1.26 & 1.57 & 1.32 \\
WS: Rmses (m s ${ }^{-1}$ ) & 0.77 & 0.92 & 0.80 \\
WS: Rmseu (m s ${ }^{-1}$ ) & 1.00 & 1.28 & 1.06 \\
WS: SYS (fraction) & 0.37 & 0.34 & 0.36 \\
WS: UNSYS (fraction) & 0.63 & 0.66 & 0.64 \\
\hline
\end{tabular}

regardless of how subtle, are important for simulating the transport and production of air pollutants through urban areas. When the mixing height and stability improvements are also considered for air-quality modeling at this scale, the UCP might be better than the roughness approach.

\section{d. Sensitivity to dynamic and thermodynamic effects of the UCP}

Sensitivities to the momentum (dynamics) and energy (thermodynamics) components of the UCP are shown in Table 4. A momentum-only case (mom_only) is run in which the changes to the energy budget due to urban areas [i.e., Eqs. (4) and (10)] are omitted. An energyonly case (en_only) is also run in which changes to the momentum and TKE [i.e., Eqs. (1)-(3)] are omitted and the roughness length is increased to $1 \mathrm{~m}$ (as in nocan 30 and nocan40). As indicated in Table 4, the full UCP case (can40) is statistically preferable to en_only and mom_only for all momentum statistics and some temperature statistics. Case en_only is generally the best for thermodynamic fields but is often inferior for momentum fields. Overall, mom_only outperforms en_only for winds, and en_only outperforms mom_only for temperature and moisture. Statistical time series (not shown) indicate that, hour by hour, can40 is statistically similar to mom_only for wind fields and is similar to en_only for temperature and moisture fields. As in Table 4, the time series indicate that en_only generally outperforms mom_only for temperature and moisture, and mom_only outperforms en_only for winds throughout the day. However, when considering all variables, can40 is overall more skillful than both sensitivity cases. This result suggests that the full UCP (as in can40 with treatment of both dynamics and thermodynamics (even using simplified approximations) is superior for urban areas at this scale, and this suggestion is consistent with the discussion reported by Rotach et al. (2002).

\section{Summary and future implications}

A UCP has been implemented into MM5 for finescale ( 1-km horizontal grid spacing) simulations of meteorological fields in the urban areas. The formulations for the UCP follow Brown and Williams (1998) and Brown (2000) by using the drag-force approach for the dynamic component and by considering radiative effects between buildings, at rooftops, and from anthropogenic sources using simplified approximations. The UCP included in MM5 is assessed for a 1.33-km domain for a real-data application in Philadelphia using the morphology of the city from Ellefsen (1991).

There are important differences between the MM5 simulations that use the UCP and the roughness length approaches, and measurements from other urban field data and wind-tunnel experiments suggest that the UCP improves model results in urban areas. In addition, comparisons with the time series of meteorological measurements indicate that the UCP simulations are better than those that use the roughness approach. In particular, the UCP tends to improve simulations of temperature, wind speed, wind direction, and PBL height in and downstream of urban areas, all of which affect air-quality modeling. The UCP can help to improve the simulations over the urban core without affecting the nonurban areas, as expected. The improvements in the UCP simulation are primarily attributed to the more explicit and suitable treatment of the urban areas rather than simply being due to the increase in the vertical resolution. The UCP overall performs best when both the dynamics and thermodynamics aspects of the parameterization are used. These results indicate that using a UCP may have significant ramifications for air-quality modeling at this scale because the dynamical characteristics of the volume in which pollutants are injected has been altered.

Further development of the UCP is warranted based on the results presented here. We recognize that strong conclusions cannot be drawn from a single case study. However, the results achieved in this initial study are promising, and applying data from one of the more recent intensive urban field campaigns (e.g., "URBAN 2000"; Allwine et al. 2002) to validate further the UCP in MM5 could be beneficial. We are currently focusing on a more sophisticated treatment of thermodynamics that can specifically consider the contributions of vegetation and the soil moisture budget following Dupont (2001) and that is reported in Dupont et al. (2003). In this next phase, we are linking the drag-force approach with an urban soil model in MM5. We also account for several of the physical processes that were neglected in this initial study, including treatment of the nonurban (i.e., rural) areas within the domain, the influence of vegetation on the canopy dynamics and thermodynamics, and modifications to the humidity equation and turbulent length scale. It is anticipated that the next phase of this work will more fully enhance our ability to sim- 
ulate the urban environment in the mesoscale model, which can also be of benefit for modeling air quality in urban areas.

Acknowledgments. The first and fourth authors were supported by EPA Interagency Agreement DW13938634-01-C with NOAA. The second and third authors were supported at the Atmospheric Sciences Modeling Division through UCAR Visiting Scientist Program appointments. The authors thank Dr. Michael Brown (Los Alamos National Laboratory) for valuable discussions regarding the formulation of the urban canopy parameterization. The authors are grateful to Dr. Richard Ellefsen (San Jose State University) for providing additional information and insight on the morphology study of Philadelphia. Valuable discussions with ASMD colleagues Drs. Daewon Byun (now at University of Houston), Jonathan Pleim, and Steven Perry also contributed to the completeness of this work. The information in this manuscript has been prepared under funding by the U.S. Environmental Protection Agency. It has been subjected to agency review and approved for publication. Mention of trade names or commercial products does not constitute endorsement or recommendation for use.

\section{APPENDIX}

\section{Statistical Measures}

The statistical measures used here are based on formulas from Willmott (1982), who describes various methods of quantifying the statistical relationships between an observed $(O)$ and model-predicted $(P)$ quantity. These statistics are computed for temperature, humidity, and vector wind difference. VWD allows for the total horizontal wind to be evaluated using a single statistic, and it is defined by Stauffer and Seaman (1990) as

$$
\mathrm{VWD}=\left[\left(U-U_{0}\right)^{2}+\left(V-V_{0}\right)^{2}\right]^{0.5},
$$

where $U$ and $V$ are the horizontal wind components and the subscript 0 refers to the observed values.

The mean absolute error, mean error (ME), and rootmean-square error provide estimates of average model error. The MAE and the ME are defined following Willmott (1982) as

$$
\begin{aligned}
\text { MAE } & =N^{-1} \sum_{i=1}^{N}\left|P_{i}-O_{i}\right| \quad \text { and } \\
\mathrm{ME} & =N^{-1} \sum_{i=1}^{N}\left(P_{i}-O_{i}\right),
\end{aligned}
$$

where $N$ is the number of observation and model pairs and $i$ is the $i$ th pair. The MAE is always positive, and it summarizes the mean difference between $P$ and $O$. The ME [called "mean bias error" in Willmott (1982)] is the difference between the mean of the model-pre- dicted variable and the mean of the observed variable $\overline{(O)}$.

The index of agreement can be used to assess relative model performance. The IA is defined in Willmott (1982) as

$$
\mathrm{IA}=1-\left[\sum_{i=1}^{N}\left(P_{i}-O_{i}\right)^{2} / \sum_{i=1}^{N}\left(\left|P_{i}^{\prime}\right|+\left|O_{i}^{\prime}\right|\right)^{2}\right],
$$

where $P_{i}^{\prime}=P_{i}-\bar{O}$ and $O_{i}^{\prime}=O_{i}-\bar{O}$. The IA is bounded between 0 and 1 such that the perfect simulation has IA $=1$.

The rmse is computed from

$$
\text { rmse }=\left[N^{-1} \sum_{i=1}^{N}\left(P_{i}-O_{i}\right)^{2}\right]^{0.5} .
$$

The rmse is always positive, and it emphasizes extreme differences between $P$ and $O$.

The systematic and unsystematic rmse (rmses and rmseu) are used to quantify the type of error. Rmses and rmseu are calculated by accounting for the slope and intercept of the regression line that compare observed and predicted values and using

$$
\begin{aligned}
& \text { rmses }=\left[N^{-1} \sum_{i=1}^{N}\left(\hat{P}_{i}-O_{i}\right)^{2}\right]^{0.5} \text { and } \\
& \text { rmseu }=\left[N^{-1} \sum_{i=1}^{N}\left(P_{i}-\hat{P}_{i}\right)^{2}\right]^{0.5},
\end{aligned}
$$

where $\hat{P}_{i}=a+b O_{i}$ such that $a$ is the intercept and $b$ is the slope of the least squares regression $\hat{P}$. Rmses and rmseu form the following relation:

$$
(\text { rmse })^{2}=(\text { rmses })^{2}+(\text { rmseu })^{2} .
$$

Note that the mean-square error (MSE) is equivalent to $(\text { rmse })^{2}$. Equation (A8) suggests that the MSE is equal to the sum of its systematic and unsystematic components. Thus, the proportion of systematic error in the model can be defined from

$$
\begin{aligned}
\text { SYS } & =\frac{\text { MSES }}{\text { MSE }} \text { and } \\
\text { UNSYS } & =\frac{\text { MSEU }}{\text { MSE }} .
\end{aligned}
$$

In theory, systematic errors should account for processes that the model does not routinely simulate well, whereas unsystematic errors could be attributed to randomness or subgrid-scale processes. Willmott (1982) states that a "good" model will have a systematic MSE that approaches 0 while the unsystematic MSE approaches the MSE. Therefore, better models should have a smaller systematic portion of the error (i.e., bias).

\section{REFERENCES}

Allwine, K. J., J. H. Shinn, G. E. Streit, K. L. Clawson, and M. Brown, 2002: Overview of URBAN 2000: A multiscale field 
study of dispersion through an urban environment. Bull. Amer. Meteor. Soc., 83, 521-536.

ASHRAE, 1967: Handbook of Fundamentals. ASHRAE, 544 pp.

Bélair, S., J. Mailhot, J. W. Strapp, and J. I. MacPherson, 1999: An examination of local versus nonlocal aspects of a TKE-based boundary layer scheme in clear convective conditions. J. Appl. Meteor., 38, 1499-1518.

Berman, S., J.-Y. Ku, and S. T. Rao, 1999: Spatial and temporal variation in the mixing depth over the northeastern United States during the summer of 1995. J. Appl. Meteor., 38, 1661-1673.

Bougeault, P., and P. Lacarrère, 1989: Parameterization of orographyinduced turbulence in a mesobeta-scale model. Mon. Wea. Rev., 117, 1872-1890.

Brown, M. J., 2000: Urban parameterizations for mesoscale meteorological models. Mesoscale Atmospheric Dispersion, Z. Boybeyi, Ed., Wessex Press, 193-255.

—_, and M. D. Williams, 1998: An urban canopy parameterization for mesoscale models. Preprints, Second Urban Environment Symp., Albuquerque, NM, Amer. Meteor. Soc., 144-147.

Burian, S. J., M. J. Brown, and S. P. Velugubantla, 2002: Building height characteristics in three U.S. cities. Preprints, Fourth Symp. on Urban Environment, Norfolk, VA, Amer. Meteor. Soc., 129130.

Byun, D. W., and S. P. S. Arya, 1986: A study of mixed layer momentum evolution. Atmos. Environ., 20, 715-728.

Chen, F., and J. Dudhia, 2001: Coupling an advanced land surfacehydrology model with the Penn State-NCAR MM5 modeling system. Part II: Preliminary model validation. Mon. Wea. Rev., 129, 587-604.

Chin, H. S., M. L. Leach, and M. J. Brown, 2000: A sensitivity study of the urban effect on a regional scale model: An idealized case. Preprints, Third Symp. on Urban Environments, Davis, CA, Amer. Meteor. Soc., 76-77.

Christen, A., R. Vogt, M. W. Rotach, and E. Parlow, 2002: First results from BUBBLE I: Profiles of fluxes in the urban roughness sublayer. Preprints, Fourth Symp. on Urban Environments, Norfolk, VA, Amer. Meteor. Soc., 105-106.

Dudhia, J., 1989: Numerical study of convection observed during the winter monsoon experiment using a mesoscale two-dimensional model. J. Atmos. Sci., 46, 3077-3107.

Dupont, S., 2001: Modélisation dynamique et thermodynamique de la canopée urbaine: Réalisation du modèle de sols urbains pour SUBMESO (Dynamic and thermodynamic modeling of the urban canopy: Development of the urban soil model for SUBMESO). $\mathrm{Ph} . D$. thesis, ECN-Université de Nantes, France, 319 pp.

_, T. Otte, A. Lacser, and J. Ching, 2003: Using MM5 to simulate the meteorological fields at neighborhood scales. Proc. Fourth Int. Conf. on Urban Air Quality: Measurements, Modelling, and Management, Prague, Czech Republic, Institute of Physics, 428431

Ellefsen, R., 1991: Mapping and measuring buildings in the canopy boundary layer in ten U.S. cities. Energy Build., 16, 1025-1049.

Grell, G., J. Dudhia, and D. R. Stauffer, 1994: A description of the Fifth-Generation Penn State/NCAR Mesoscale Model (MM5). NCAR Tech. Note NCAR/TN-398+STR, 138 pp.

Grimmond, C. S. B., and T. R. Oke, 1995: Comparison of heat fluxes from summertime observations in the suburbs of four North American cities. J. Appl. Meteor., 34, 873-889.

_ , and —_, 1999a: Heat storage in urban areas: Local-scale observations and evaluation of a simple model. J. Appl. Meteor., 38, 922-940.

— rived from analysis of surface form. J. Appl. Meteor., 38, 1262 1292.

Kastner-Klein, P., E. Fedorovich, and M. W. Rotach, 2001: A wind tunnel study of organised and turbulent air motions in urban street canyons. J. Wind. Eng. Ind. Aerodyn., 89, 849-861.

Martilli, A., 2002: Numerical study of urban impact on boundary layer structure: Sensitivity to wind speed, urban morphology, and rural soil moisture. J. Appl. Meteor., 41, 1247-1266.
- A. Clappier, and M. W. Rotach, 2002: An urban surface exchange parameterisation for mesoscale models. Bound.-Layer Meteor., 104, 261-304.

Maruyama, T., 1999: Surface and inlet boundary conditions for the simulation of turbulent boundary layer over complex rough surfaces. J. Wind. Eng. Ind. Aerodyn., 81, 311-322.

Masson, V., 2000: A physically-based scheme for the urban energy budget. Bound.-Layer Meteor., 94, 357-397.

Mlawer, E. J., S. J. Taubman, P. D. Brown, M. J. Iacono, and S. A. Clough, 1997: Radiative transfer for inhomogeneous atmospheres: RRTM, a validated correlated- $k$ model for the longwave. J. Geophys. Res., 102, $16663-16682$.

Oke, T. R., 1995: The heat island of the urban boundary layer: Characteristics, causes and effects. Wind Climate in Cities, J. E. Cermak et al., Eds., Kluwer Academic, 81-107.

Pielke, R. A., 1984: Mesoscale Meteorological Modeling. Academic Press, $612 \mathrm{pp}$

Ratti, C., S. Di Sabatino, R. E. Britter, M. J. Brown, F. Caton, and S. Burian, 2002: Analysis of 3-D urban databases with respect to pollution dispersion for a number of European and American cities. Water Air Soil Pollut. Focus, 2, 459-469.

Reisner, J., R. J. Rasmussen, and R. T. Bruintjes, 1998: Explicit forecasting of supercooled liquid water in winter storms using the MM5 mesoscale model. Quart. J. Roy. Meteor. Soc., 124B, 1071-1107.

Rotach, M. W., 1999: On the influence of the urban roughness sublayer on turbulence and dispersion. Atmos. Environ., 33, 40014008

_ 2001 : Simulation of urban-scale dispersion using a Lagrangian stochastic dispersion model. Bound.-Layer Meteor., 99, 379410.

_ B. Fisher, and M. Piringer, 2002: COST 715 workshop on urban boundary layer parameterizations. Bull. Amer. Meteor. Soc., 83, $1501-1504$.

Roth, M., 2000: Review of atmospheric turbulence over cities. Quart. J. Roy. Meteor. Soc., 126, 941-990.

Sailor, D. J., 1995: Simulated urban climate response to modifications in surface albedo and vegetative cover. J. Appl. Meteor., 34, $1694-1704$.

- and H. Fan, 2002: Modeling the diurnal variability of effective albedo for cities. Atmos. Environ., 36, 713-725.

Seaman, N. L., and S. A. Michelson, 2000: Mesoscale meteorological structure of a high-ozone episode during the 1995 NARSTONortheast study. J. Appl. Meteor., 39, 384-398.

Shafran, P. C., N. L. Seaman, and G. A. Gayno, 2000: Evaluation of numerical predictions of boundary layer structure during the Lake Michigan Ozone Study. J. Appl. Meteor., 39, 412-426.

Stauffer, D. R., and N. L. Seaman, 1990: Use of four-dimensional data assimilation in a limited-area mesoscale model. Part I: Experiments with synoptic-scale data. Mon. Wea. Rev., 118, 1250 1277.

- and _ 1994: Multiscale four-dimensional data assimilation. J. Appl. Meteor., 33, 416-434.

Taha, H., 1999: Modifying a mesoscale model to better incorporate urban heat storage: A bulk parameterization approach. J. Appl. Meteor., 38, 466-473.

Uno, I., H. Ueda, and S. Wakamatsu, 1989: Numerical modeling of the nocturnal urban boundary layer. Bound.-Layer Meteor., 49, 77-98.

Voogt, J. A., and C. S. B. Grimmond, 2000: Modeling surface sensible heat flux using surface radiative temperatures in a simple urban area. J. Appl. Meteor., 39, 1679-1699.

Vu, T. C., Y. Ashie, and T. Asaeda, 2002: A $k-\varepsilon$ turbulence closure model for the atmospheric boundary layer including urban canopy. Bound.-Layer Meteor., 102, 459-490.

Willmott, C. J., 1982: Some comments on the evaluation of model performance. Bull. Amer. Meteor. Soc., 63, 1309-1313.

Xiu, A., and J. E. Pleim, 2001: Development of a land surface model. 
Part I: Application in a mesoscale meteorological model. J. Appl. Meteor., 40, 192-209.

Yamada, T., 1982: A numerical model study of turbulent airflow in and above a forest canopy. J. Meteor. Soc., Japan, 60, 439-454.

Zhang, D.-L., and R. A. Anthes, 1982: A high-resolution model of the planetary boundary layer-Sensitivity tests and comparisons with SESAME-79 data. J. Appl. Meteor., 21, 1594-1609.

Zhang, K., and Coauthors, 2001: Numerical investigation of boundary-layer evolution and nocturnal low-level jets: Local versus non-local PBL schemes. Environ. Fluid Mech., 1, 171-208. 